\title{
Numerical Study of Switching Behavior in Finite Media Subject to 3D Ferroelectric-Paraelectric Interactions and Inspection of Calibration Effects
}

\author{
P.-W. Martelli* and S.M. Mefire \\ Université de Lorraine, CNRS, IECL, F-54000 Nancy, France
}

Received 31 August 2019; Accepted (in revised version) 10 May 2020

\begin{abstract}
We study numerically the switching behavior aspects and calibration effects relative to finite media embedding fully a three-dimensional ferroelectric layer in a paraelectric environment. Our approach makes use of the Ginzburg-Landau formalism in combination with the electrostatics equations. The associated discrete nonlinear system, which arises from finite element approximations, is solved by an inexact Newton method. The resulting numerical experiments highlight the effects of a balance between the physical and geometrical parameters. In particular, the same state switchings can be retrieved from different ferroelectric layer sizes by acting upon the physical characteristic of the paraelectric environment. Ferroelectric platelet samples are in parallelepipedic and cylindrical configurations involved in these experiments.
\end{abstract}

AMS subject classifications: 82D45, 82D80, 78A25, 35Q56, 47H10, 65N30, 49M15

Key words: Electroactive media, ferroelectricity, electrostatics, Ginzburg-Landau systems, finite elements, inexact Newton methods, numerical simulations.

\section{Introduction}

For performing a relevant numerical investigation of structures comprised of a ferroelectric layer embedded in a paraelectric environment, a method involving a description as accurate as possible of the interplay between the spontaneous polarization and the long-range depolarization electric field caused by the same polarization is particularly suitable. For a finite-size sample, the depolarization field is distributed in the inner space and in the surrounding environment at the cost of an additional electrostatic energy. A notable property is that, even in the absence of the application of an external field, this inner space can be organized into a finite number of distinct regions, called the domains, in which the electric polarization is arranged uniformly [18]. Namely, the

\footnotetext{
${ }^{*}$ Corresponding author. Email address: pierre-william.martelli@ac-nancy-metz.fr (P.-W. Martelli) 
depolarization electric field splits the finite-size ferroelectric sample into polarization domains.

Of course, when an external field is applied, the polarization domain patterning is subject to a complex behavior; the system is then characterized by various ferroelectric states. Recently, a work has been developed [13] to explicit how the Landau-Kittel structure [7-9] of $180^{\circ}$ polarization domains is formed in finite-scale nanodot samples. In particular, it is highlighted in [13] that field and temperature applications allow to realize a controllable multibit switching; an effect leading thus to the ability to increase the volume of the writable information per nanodot. The approach used in [13] was introduced by Chenskii and Tarasenko [3]. It consists of completing the model of Ginzburg [5] that was based on the theory of phase transitions developed by Landau [9], with the electrostatics equations, in order to take into account long-range Coulomb interactions. This method, particularly suited to an accurate description of the interplay aforementioned, has been used also in a two-dimensional context involving periodic boundary conditions [10]. Moreover, it has been applied in the frame of certain threedimensional ferroelectric devices $[12,13]$.

We deal here with this approach, and to dissociate the depolarization effects from the lattice deformation impacts, we consider uniaxial ferroelectric materials for which the ferroelastic coupling is small. In particular, in presence of such a material, the dependence of the electric polarization field on the electric field is namely nonlinear through only one of its components. Unlike in [13], where the simulations were associated with a cylindrical configuration and without an investigation of the influence of geometrical parameters, we are concerned with numerical variations of geometrical and physical parameters, moreover with the parallelepipedic configuration context. In contrast with [12], where is also presented the model related to the mentioned approach, the interest is here devoted on the one hand to considerations that are above all concrete as regards temperature applications too and on the other hand to a study of calibration effects. This interest leads us to analyze here the influence of physical and geometrical parameters, then both under concrete considerations of voltage potential and temperature applications. The present work deals thus with extensive numerical investigations for which the associated findings would aim at impregnating physical experiments. In particular, reference is made here to physical experiments that could be carried out by selecting, for instance, Sodium Nitrite $\left(\mathrm{NaNO}_{2}\right)$ as the model material for the ferroelectric layer [17].

This paper is subdivided in five sections. We consider in Section 2 a weak formulation based on the Ginzburg-Landau formalism and electrostatics system, suitable for uniaxial ferroelectric materials. In Section 3, we present the main algorithm that is used for solving the discrete nonlinear system deriving from finite element discretizations of the weak formulation. This is an iterative algorithm that combines two inexact Newton techniques. The first technique is an approach globalized with a linesearch method [15], which slowly converges for a large choice of initializations, whereas the second, a more standard one, converges faster but for a restricted choice of initializations. In Section 4, we develop an extensive study of the switching behavior aspects 
and calibration effects relative to finite media embedding fully a ferroelectric layer in a paraelectric environment. Platelet samples in parallelepipedic and cylindrical configurations are considered. This study is achieved with respect to the temperature as well as in terms of the external electric field, under variations of geometrical and physical parameters. We report in Section 5 concluding remarks and perspectives.

\section{Weak formulation based on the Ginzburg-Landau formalism and electrostatics equations}

We consider the weak formulation introduced in $[11,12]$ and associated with the model presented also therein for the analysis of three-dimensional devices made up of a ferroelectric layer that is fully embedded in a paraelectric environment. Let us represent geometrically such a device with the help of an open bounded subset $\Omega$ of $\mathbb{R}^{3}$, and its fully embedded layer by an open subset $\Omega_{f}, \overline{\Omega_{f}} \subset \Omega$, as well as its paraelectric environment by $\Omega_{p}=\Omega \backslash \overline{\Omega_{f}}$. Also, let us denote by $S, S=\partial \Omega$, the boundary of $\Omega$, and by $S_{f}, S_{f}=\overline{\Omega_{f}} \cap \overline{\Omega_{p}}$, the interface between $\Omega_{f}$ and $\Omega_{p}$, as well as by $\nu=\left(\nu_{x}, \nu_{y}, \nu_{z}\right)^{T}$ the outward unit normal to $\Omega_{f}$, defined on $S_{f}$, where the superscript " $T$ " indicates the transpose here and in the next sections.

The model was based on the Ginzburg-Landau formalism in combination with the electrostatics equations. It consists of finding $P: \Omega_{f} \rightarrow \mathbb{R}$ and $\varphi: \Omega \rightarrow \mathbb{R}$ such that

$$
(E G L) \begin{cases}t P+\left(\frac{P}{P_{0}}\right)^{2} P-\nabla \cdot(\xi \nabla P)+\frac{\varkappa \|}{4 \pi} \partial_{z} \varphi=0 & \text { in } \Omega_{f}, \\ -\nabla \cdot(\varepsilon \nabla \varphi)+4 \pi \partial_{z} P=0 & \text { in } \Omega_{f}, \\ -\nabla \cdot(\varepsilon \nabla \varphi)=0 & \text { in } \Omega_{p}, \\ \left.\varphi\right|_{\Omega_{f}}=\left.\varphi\right|_{\Omega_{p}} & \text { on } S_{f}, \\ \nu \cdot\left(\left.(\varepsilon \nabla \varphi)\right|_{\Omega_{f}}-\left.(\varepsilon \nabla \varphi)\right|_{\Omega_{p}}\right)-4 \pi \nu_{z} P=0 & \text { on } S_{f}, \\ \nu \cdot(\xi \nabla P)=0 & \text { on } S_{f}, \\ \varphi=\varphi_{S} & \text { on } S,\end{cases}
$$

where

- $t$ is the reduced temperature, expressed via the Curie temperature $T_{0}$ and the temperature $T$ of the device, $t=\left(T-T_{0}\right) / T_{0} \in[-1,0)$;

- $\varkappa_{\|}$is the displacive parameter (namely positive);

- $P_{0}$ is the spontaneous polarization at low temperatures, $P_{0} \neq 0$;

- $\varepsilon$ is the permittivity tensor, considered as being symmetric;

- $\xi$ is the coherence length tensor, also considered as being symmetric;

- $\varphi_{S}$ is a given function. 
Called in the sequel, as in [12], the Electrostatic Ginzburg-Landau system and more simply (EGL), this model is related to uniaxial ferroelectric devices; namely, the ones for which the dependence of the electric polarization field, $\mathbf{P}$, on the electric field, $\mathbf{E}$, is nonlinear through only one of its components (which is here the third one). More precisely, we have

$$
\mathbf{P}=\frac{1}{4 \pi}(\varepsilon \mathbf{E}-\mathbf{E})+\left(\begin{array}{c}
0 \\
0 \\
P
\end{array}\right) \quad \text { in } \Omega_{f} .
$$

The electric field derives from the scalar potential $\varphi$; namely, $\mathbf{E}=-\nabla \varphi$.

The Eq. (2.1) involves the Ginzburg-Landau theory of phase transitions (recalling that two phases, the paraelectric phase and the ferroelectric phase, are in association with a ferroelectric body [8]). The relations (2.2) and (2.3) correspond to the electrostatics equation $\nabla \cdot \mathbf{D}=0$ expressed in $\Omega_{f}$ and $\Omega_{p}$ respectively, where $\mathbf{D}$ is the electric displacement field

$$
\mathbf{D}= \begin{cases}\varepsilon \mathbf{E}+\left(\begin{array}{c}
0 \\
0 \\
4 \pi P
\end{array}\right) & \text { in } \Omega_{f}, \\
\varepsilon \mathbf{E} & \text { in } \Omega_{p} .\end{cases}
$$

At the ferroelectric/paraelectric interface $S_{f}$, the scalar potential $\varphi$ satisfies the continuity condition (2.4), and the jump of the normal trace of $\mathbf{D}$ is equal to zero as reports (2.5). Also, the scalar field $P$ is subject to a Neumann condition and $\varphi$ satisfies a Dirichlet condition as relate (2.6) and (2.7), respectively.

We assume that each of the open subsets of $\mathbb{R}^{3}$ that are $\Omega_{f}$ and $\Omega$ has a Lipschitzcontinuous boundary. Also, we consider the tensors $\xi$ and $\varepsilon$, where

$$
\xi=\left(\xi_{i, j}\right)_{1 \leq i, j \leq 3}, \quad \varepsilon=\left(\varepsilon_{i, j}\right)_{1 \leq i, j \leq 3},
$$

in such a way that

$$
\begin{aligned}
& \forall 1 \leq i, j \leq 3, \quad \xi_{i, j} \in L^{\infty}\left(\Omega_{f}\right), \quad \varepsilon_{i, j} \in L^{\infty}(\Omega), \\
& \exists \xi_{0}>0 \quad \forall d=\left(d_{1}, d_{2}, d_{3}\right)^{T} \in \mathbb{R}^{3}, \quad \sum_{i, j=1}^{3} \xi_{i, j} d_{j} d_{i} \geq \xi_{0} \sum_{i=1}^{3} d_{i}^{2} \quad \text { a.e. } \quad \text { in } \Omega_{f}, \\
& \exists \varepsilon_{0}>0 \quad \forall d=\left(d_{1}, d_{2}, d_{3}\right)^{T} \in \mathbb{R}^{3}, \quad \sum_{i, j=1}^{3} \varepsilon_{i, j} d_{j} d_{i} \geq \varepsilon_{0} \sum_{i=1}^{3} d_{i}^{2} \quad \text { a.e. in } \Omega .
\end{aligned}
$$

We set

$$
W=H^{1}\left(\Omega_{f}\right) \times H_{0}^{1}(\Omega),
$$

and represent by $\|\cdot\|_{W}$ the norm defined on $W$, where

$$
\|(P, \varphi)\|_{W}=\left(\|P\|_{H^{1}\left(\Omega_{f}\right)}^{2}+|\varphi|_{H^{1}(\Omega)}^{2}\right)^{\frac{1}{2}} \text { for }(P, \varphi) \in W .
$$


As introduced in [12], with more details in [11], the weak formulation associated with (EGL) consists of finding $(P, \varphi) \in W$ such that for $(Q, \psi) \in W$

$$
\left\{\begin{aligned}
& \int_{\Omega_{f}} t P Q \mathrm{dx}+\int_{\Omega_{f}} \frac{P^{3}}{P_{0}^{2}} Q \mathrm{dx}+\int_{\Omega_{f}}(\xi \nabla P) \cdot \nabla Q \mathrm{dx}+\int_{\Omega_{f}} \frac{\varkappa \|}{4 \pi}\left(\partial_{z} \varphi\right) Q \mathrm{dx} \\
= & -\int_{\Omega_{f}} \frac{\varkappa \|}{4 \pi}\left(\partial_{z} w\right) Q \mathrm{dx}, \\
& \int_{\Omega}(\varepsilon \nabla \varphi) \cdot \nabla \psi \mathrm{dx}-\int_{S_{f}} 4 \pi \nu_{z} P \psi \mathrm{d} \sigma+\int_{\Omega_{f}} 4 \pi\left(\partial_{z} P\right) \psi \mathrm{dx} \\
= & -\int_{\Omega}(\varepsilon \nabla w) \cdot \nabla \psi \mathrm{dx}
\end{aligned}\right.
$$

with $w$ considered in such a way that $w \in H^{1}(\Omega)$ and $\left.w\right|_{S}=\varphi_{S}$, where $\varphi_{S} \in H^{\frac{1}{2}}(S)$.

Proposition 2.1. Let us consider $t \leq 0, P_{0} \neq 0, \varkappa_{\|} \geq 0$, as well as $\xi$ and $\varepsilon$ subject to (2.9)-(2.11). Also, let $w \in H^{1}(\Omega)$ be defined as above. Then, if $(P, \varphi) \in W$ satisfies the weak formulation (2.12) and (2.13), it follows that

$$
\begin{cases}t P+\frac{P^{3}}{P_{0}^{2}}-\nabla \cdot(\xi \nabla P)+\frac{\varkappa_{\|}}{4 \pi} \partial_{z}(\varphi+w)=0 & \text { in } L^{2}\left(\Omega_{f}\right), \\ -\nabla \cdot(\varepsilon \nabla(\varphi+w))+4 \pi \partial_{z} P=0 & \text { in } L^{2}\left(\Omega_{f}\right), \\ -\nabla \cdot(\varepsilon \nabla(\varphi+w))=0 & \text { in } L^{2}\left(\Omega_{p}\right) .\end{cases}
$$

Proof. Let $(P, \varphi) \in W$ satisfy (2.12) and (2.13), under the considered assumptions. Then it follows from [6] that $P^{3} \in L^{2}\left(\Omega_{f}\right)$. By denoting by $\langle\cdot, \cdot\rangle$ the duality pairing between the dual space $\mathcal{D}^{\prime}\left(\Omega_{f}\right)$ and $\mathcal{D}\left(\Omega_{f}\right)$, we obtain from (2.12), with the notation $\mathrm{x}=(x, y, z)=:\left(x_{1}, x_{2}, x_{3}\right)$ and for all $Q \in \mathcal{D}\left(\Omega_{f}\right)$, that

$$
\left\langle t P+\frac{P^{3}}{P_{0}^{2}}, Q\right\rangle+\sum_{i=1}^{3}\left\langle\sum_{j=1}^{3} \xi_{i, j} \partial_{x_{j}} P, \partial_{x_{i}} Q\right\rangle+\left\langle\frac{\varkappa_{\|}}{4 \pi} \partial_{z} \varphi, Q\right\rangle=-\left\langle\frac{\varkappa_{\|}}{4 \pi} \partial_{z} w, Q\right\rangle
$$

i.e.,

$$
\left\langle t P+\frac{P^{3}}{P_{0}^{2}}-\sum_{i, j=1}^{3} \partial_{x_{i}}\left(\xi_{i, j} \partial_{x_{j}} P\right)+\frac{\varkappa_{\|}}{4 \pi} \partial_{z} \varphi, Q\right\rangle=-\left\langle\frac{\varkappa_{\|}}{4 \pi} \partial_{z} w, Q\right\rangle
$$

Thus,

$$
t P+\frac{P^{3}}{P_{0}^{2}}-\nabla \cdot(\xi \nabla P)+\frac{\varkappa_{\|}}{4 \pi} \partial_{z} \varphi=-\frac{\varkappa_{\|}}{4 \pi} \partial_{z} w \quad \text { in } \mathcal{D}^{\prime}\left(\Omega_{f}\right),
$$

and it follows then (2.14).

Also, with the same assumptions and notation, we obtain from (2.13), and for all $\psi$ with $\left.\psi\right|_{\Omega_{f}} \in \mathcal{D}\left(\Omega_{f}\right),\left.\psi\right|_{\Omega_{p}}=0$, that:

$$
\sum_{i=1}^{3}\left\langle\sum_{j=1}^{3} \varepsilon_{i, j} \partial_{x_{j}} \varphi, \partial_{x_{i}} \psi\right\rangle+\left\langle 4 \pi \partial_{z} P, \psi\right\rangle=-\sum_{i=1}^{3}\left\langle\sum_{j=1}^{3} \varepsilon_{i, j} \partial_{x_{j}} w, \partial_{x_{i}} \psi\right\rangle,
$$


i.e.,

$$
\left\langle-\sum_{i, j=1}^{3} \partial_{x_{i}}\left(\varepsilon_{i, j} \partial_{x_{j}} \varphi\right)+4 \pi \partial_{z} P, \psi\right\rangle=\left\langle\sum_{i, j=1}^{3} \partial_{x_{i}}\left(\varepsilon_{i, j} \partial_{x_{j}} w\right), \psi\right\rangle
$$

Thus,

$$
-\nabla \cdot(\varepsilon \nabla(\varphi+w))+4 \pi \partial_{z} P=0 \quad \text { in } \mathcal{D}^{\prime}\left(\Omega_{f}\right)
$$

and we get (2.15).

In the same way, under these assumptions and by considering now $\psi$ with $\left.\psi\right|_{\Omega_{p}} \in$ $\mathcal{D}\left(\Omega_{p}\right),\left.\psi\right|_{\Omega_{f}}=0$, in (2.13), it derives then (2.16).

Remark 2.1. If with the assumptions of Proposition 2.1 considering moreover the coefficients of the tensors $\xi$ and $\varepsilon$ as being regular, as well with $w$ more regular, we have that $(P, \varphi) \in W$ satisfies (2.12) and (2.13), and $P \in H^{2}\left(\Omega_{f}\right), \xi_{i, j} \partial_{a} P \in H^{1}\left(\Omega_{f}\right)$ for $1 \leq i, j, a \leq 3$, where $\left(\partial_{1}, \partial_{2}, \partial_{3}\right):=\left(\partial_{x}, \partial_{y}, \partial_{z}\right)$, it derives then the relation below. Namely, we get in this case, by using the Green formula from (2.12) and for all $Q \in H^{1}\left(\Omega_{f}\right)$, that

$$
\begin{aligned}
\int_{\Omega_{f}} t P Q \mathrm{dx} & +\int_{\Omega_{f}} \frac{P^{3}}{P_{0}^{2}} Q \mathrm{dx}-\int_{\Omega_{f}} \nabla \cdot(\xi \nabla P) Q \mathrm{dx}+\int_{S_{f}} \nu \cdot(\xi \nabla P) Q \mathrm{~d} \sigma \\
& +\int_{\Omega_{f}} \frac{\varkappa \|}{4 \pi}\left(\partial_{z} \varphi\right) Q \mathrm{dx}=-\int_{\Omega_{f}} \frac{\varkappa \|}{4 \pi}\left(\partial_{z} w\right) Q \mathrm{dx} .
\end{aligned}
$$

By taking into account (2.14), it follows that

$$
\int_{S_{f}} \nu \cdot(\xi \nabla P) Q \mathrm{~d} \sigma=0 \quad \text { for all } \quad Q \in H^{1}\left(\Omega_{f}\right)
$$

It results then, under regularity of $S_{f}$ also, that in this case $\nu \cdot(\xi \nabla P)=0$ in $L^{2}\left(S_{f}\right)$.

Let us also mention that if, under the previous assumptions, $(P, \varphi) \in W$ satisfies (2.12) and (2.13), and $\left.(\varphi+w)\right|_{\Omega_{f}} \in H^{2}\left(\Omega_{f}\right),\left.(\varphi+w)\right|_{\Omega_{p}} \in H^{2}\left(\Omega_{p}\right),\left.\left(\varepsilon_{i, j} \partial_{a}(\varphi+w)\right)\right|_{\Omega_{f}} \in$ $H^{1}\left(\Omega_{f}\right),\left.\left(\varepsilon_{i, j} \partial_{a}(\varphi+w)\right)\right|_{\Omega_{p}} \in H^{1}\left(\Omega_{p}\right)$, then $(P, \phi):=(P, \varphi+w)$ is subject to (2.5) in the following weak sense. Namely, we obtain from (2.13), and by using the Green formula, that for all $\psi \in H_{0}^{1}(\Omega)$

$$
\begin{aligned}
& -\int_{\Omega_{f}} \nabla \cdot(\varepsilon \nabla(\varphi+w)) \psi \mathrm{dx}+\left.\int_{S_{f}} \nu \cdot(\varepsilon \nabla(\varphi+w))\right|_{\Omega_{f}} \psi \mathrm{d} \sigma-\int_{S_{f}} 4 \pi \nu_{z} P \psi \mathrm{d} \sigma \\
& \quad+\int_{\Omega_{f}} 4 \pi\left(\partial_{z} P\right) \psi \mathrm{dx}-\int_{\Omega_{p}} \nabla \cdot(\varepsilon \nabla(\varphi+w)) \psi \mathrm{dx}-\left.\int_{S_{f}} \nu \cdot(\varepsilon \nabla(\varphi+w))\right|_{\Omega_{p}} \psi \mathrm{d} \sigma=0 .
\end{aligned}
$$

It follows then, by using (2.15) and (2.16), that

$$
\int_{S_{f}}\left(\nu \cdot\left(\left.(\varepsilon \nabla \phi)\right|_{\Omega_{f}}-\left.(\varepsilon \nabla \phi)\right|_{\Omega_{p}}\right)-4 \pi \nu_{z} P\right) \psi \mathrm{d} \sigma=0 \quad \text { for all } \quad \psi \in H_{0}^{1}(\Omega) .
$$


Although in the applications the temperature $T$ takes values in such a way that $t \in$ $[-1,0)$, the statement below considers a broader context of this parameter. Established in [12], this statement reports the existence of solutions of the formulation.

Theorem 2.1. Let us consider $t \leq 0, P_{0} \neq 0, \varkappa_{\|} \geq 0$, as well as $\xi$ and $\varepsilon$ subject to (2.9)(2.11). Furthermore, let $\varphi_{S} \in H^{\frac{1}{2}}(S)$ and $w \in H^{1}(\Omega)$ such that $\left.w\right|_{S}=\varphi_{S}$. Then, there exists a solution of the formulation (2.12) and (2.13).

Let us note that if $(P, \varphi)$ verifies (2.12) and (2.13), then it follows that $(P, \varphi+w)$ is a weak solution of (EGL), and the (weak) electric polarization field $\mathbf{P}$ is determined from (2.8), wherein $\mathbf{E}=-\nabla(\varphi+w)$.

As specified also in [12], there is not a uniqueness of solution of the formulation for any choice of the parameters. Namely for instance, if $t<0, P_{0} \neq 0, \varkappa_{\|}=0$, and $\varphi_{S}=0$ (as well as $w=0$ ), then two distinct pairs $(P, \varphi),(-P,-\varphi)$, where $P:=\sqrt{|t|}\left|P_{0}\right|$, satisfy (2.12) and (2.13); in addition to the fact that the pair $(0,0)$ is already subject to (2.12) and (2.13) with these considerations.

\section{Discretizations and algorithms}

\subsection{Discrete nonlinear system}

We express here the nonlinear matrix system that arises from the discrete formulation associated with (2.12) and (2.13). Namely, $\Omega_{f}$ and $\Omega$ are considered as being polyhedral. The discretizations, achieved as in [12], make use of a mesh of $\bar{\Omega}$ and of Lagrange finite elements. Such a mesh consists of a collection of tetrahedra, $\mathcal{T}_{h}$, resulting from a usual process of triangulation. In particular, for the non obvious geometrical shapes, this mesh is fine enough near the boundaries of $\Omega_{f}$ and $\Omega$. The interface $S_{f}$ is entirely made up of faces of tetrahedra. Each of these faces is common both to a tetrahedron contained in $\overline{\Omega_{f}}$ and to another one included in the environment $\overline{\Omega_{p}}$. By denoting by $h$ the mesh size of $\bar{\Omega}$, let us consider the discrete space $W_{h}$

$$
W_{h}=X_{h} \times Y_{h}
$$

with

$$
\begin{aligned}
X_{h} & =\left\{P_{h} \in C^{0}\left(\overline{\Omega_{f}}\right) ;\left.P_{h}\right|_{T} \in P_{1, T} \forall T \in \mathcal{T}_{h}, T \subset \overline{\Omega_{f}}\right\}, \\
Y_{h} & =\left\{\varphi_{h} \in C^{0}(\bar{\Omega}) ;\left.\varphi_{h}\right|_{T} \in P_{1, T} \forall T \in \mathcal{T}_{h},\left.\varphi_{h}\right|_{S}=0\right\},
\end{aligned}
$$

and $P_{1, T}$ the space of polynomials of degree less than or equal to 1 , defined on $T$.

The discrete formulation associated with (2.12) and (2.13) consists of finding $\left(P_{h}, \varphi_{h}\right) \in W_{h}$ such that for all $\left(Q_{h}, \psi_{h}\right) \in W_{h}$ 


$$
\left\{\begin{aligned}
& \int_{\Omega_{f}} t P_{h} Q_{h} \mathrm{dx}+\int_{\Omega_{f}} \frac{P_{h}^{3}}{P_{0}^{2}} Q_{h} \mathrm{dx}+\int_{\Omega_{f}}\left(\xi \nabla P_{h}\right) \cdot \nabla Q_{h} \mathrm{dx}+\int_{\Omega_{f}} \frac{\varkappa \|}{4 \pi}\left(\partial_{z} \varphi_{h}\right) Q_{h} \mathrm{dx} \\
= & -\int_{\Omega_{f}} \frac{\varkappa \|}{4 \pi}\left(\partial_{z} w\right) Q_{h} \mathrm{dx}, \\
& \int_{\Omega}\left(\varepsilon \nabla \varphi_{h}\right) \cdot \nabla \psi_{h} \mathrm{dx}-\int_{S_{f}} 4 \pi \nu_{z} P_{h} \psi_{h} \mathrm{~d} \sigma+\int_{\Omega_{f}} 4 \pi\left(\partial_{z} P_{h}\right) \psi_{h} \mathrm{dx} \\
= & -\int_{\Omega}(\varepsilon \nabla w) \cdot \nabla \psi_{h} \mathrm{dx} .
\end{aligned}\right.
$$

Conforming discretizations of the regions $\overline{\Omega_{f}}$ and $\bar{\Omega}$, as well as a conforming discretization of the space $W$, are thus used; namely, $W_{h} \subset W$. By considering then the data $t$, $P_{0}, \varkappa_{\|}, \xi$, $\varepsilon$, and $w$ related to $\varphi_{S}$, under the assumptions of Theorem 2.1, it arises that the discrete formulation (3.1) has at least one solution. Let us specify that these data will be so considered in what follows and in the next section. Also, as it was reported for the case of the continuous formulation, let us indicate that there is not a uniqueness of solution of the discrete formulation for any choice of data under these same assumptions. By dealing with any solution $\left(P_{h}, \varphi_{h}\right)$ of this discrete formulation, we determine the resulting discrete electric polarization in association with $\mathbf{P}$ by using (2.8), wherein $P$ is replaced by $P_{h}$ and $\mathbf{E}$ by $-\nabla\left(\varphi_{h}+w\right)$.

Let us denote by $\left\{\mathrm{x}_{j} ; 1 \leq j \leq N\right\}$ the set of vertices of $\mathcal{T}_{h}$, numbered in such a way that $\mathrm{x}_{1}, \ldots, \mathrm{x}_{N_{f}} \in \overline{\Omega_{f}}$ and $\mathrm{x}_{1}, \ldots, \mathrm{x}_{N^{0}} \in \Omega$, where $N_{f}, N^{0}$ and $N$ are respectively the number of vertices in $\overline{\Omega_{f}}, \Omega$ and $\bar{\Omega}$; namely, $N_{f}<N^{0}<N$. In what follows, $\llbracket j_{1}, j_{2} \rrbracket$ represents, with $j_{1}, j_{2} \in \mathbb{N}$ and $j_{1}<j_{2}$, the set of integers $j$ such that: $j_{1} \leq j \leq j_{2}$. Let $u_{j}$ be the shape function associated with $\mathrm{x}_{j}$, for $j \in \llbracket 1, N \rrbracket$. We have thus, for $\left(P_{h}, \varphi_{h}\right) \in W_{h}$,

$$
P_{h}=\sum_{j=1}^{N_{f}} P_{j} u_{j}, \quad \varphi_{h}=\sum_{j=1}^{N^{0}} \varphi_{j} u_{j},
$$

where $P_{j} \in \mathbb{R}$, for all $j \in \llbracket 1, N_{f} \rrbracket$, and $\varphi_{j} \in \mathbb{R}$, for all $j \in \llbracket 1, N^{0} \rrbracket$. The discrete formulation (3.1) can then be written as a nonlinear matrix system having two vector unknowns. It consists of finding $\mathcal{P}=\left(P_{1}, \ldots, P_{N_{f}}\right)^{T} \in \mathbb{R}^{N_{f}}$ and $\Phi=\left(\varphi_{1}, \ldots, \varphi_{N^{0}}\right)^{T} \in$ $\mathbb{R}^{N^{0}}$ satisfying

$$
\left\{\begin{array}{l}
A \mathcal{P}+\mathcal{N}(\mathcal{P})+B \Phi=F_{1}, \\
C \mathcal{P}+D \Phi=F_{2}
\end{array}\right.
$$

where

$$
\begin{aligned}
& A=\left(\int_{\Omega_{f}}\left(t u_{j} u_{i}+\left(\xi \nabla u_{j}\right) \cdot \nabla u_{i}\right) \mathrm{dx}\right)_{(i, j) \in \llbracket 1, N_{f} \rrbracket^{2}}, \\
& B=\left(\int_{\Omega_{f}} \frac{\varkappa \|}{4 \pi}\left(\partial_{z} u_{j}\right) u_{i} \mathrm{dx}\right)_{(i, j) \in \llbracket 1, N_{f} \rrbracket \times \llbracket 1, N^{0} \rrbracket},
\end{aligned}
$$




$$
\begin{aligned}
& C=\left(-\int_{S_{f}} 4 \pi \nu_{z} u_{j} u_{i} \mathrm{~d} \sigma+\int_{\Omega_{f}} 4 \pi\left(\partial_{z} u_{j}\right) u_{i} \mathrm{dx}\right)_{(i, j) \in \llbracket 1, N^{0} \rrbracket \times \llbracket 1, N_{f} \rrbracket}, \\
& D=\left(\int_{\Omega}\left(\varepsilon \nabla u_{j}\right) \cdot \nabla u_{i} \mathrm{dx}\right)_{(i, j) \in \llbracket 1, N^{0} \rrbracket^{2}}, \\
& \mathcal{N}(\mathcal{P})=\left(\int_{\Omega_{f}} \frac{1}{P_{0}^{2}}\left(\sum_{j=1}^{N_{f}} P_{j} u_{j}\right)^{3} u_{i} \mathrm{dx}\right)_{i \in \llbracket 1, N_{f} \rrbracket}, \\
& F_{1}=\left(-\int_{\Omega_{f}} \frac{\varkappa \|}{4 \pi}\left(\partial_{z} w\right) u_{i} \mathrm{dx}\right)_{i \in \llbracket 1, N_{f} \rrbracket}, \\
& F_{2}=\left(-\int_{\Omega}(\varepsilon \nabla w) \cdot \nabla u_{i} \mathrm{dx}\right)_{i \in \llbracket 1, N^{0} \rrbracket} .
\end{aligned}
$$

Let us mention already that, in the frame of numerical computations regarding the experiments that will be involved in the next section, the terms of these blocks are evaluated with the help of a 5-th order quadrature formula [20]. Of course, when the tensors $\varepsilon$ and $\xi$ are constant in the geometrical elements and if $w$ is in particular a polynomial of degree less than or equal to 1 , this becomes then an exact formula in the associated evaluations. It will always be the case in the next section since $\varepsilon$ and $\xi$ will be so used, as well as $w$ from the considerations of $\varphi_{S}$, in accordance of course with the applications.

\subsection{Iterative algorithms}

In order to solve the nonlinear matrix system (3.2), we consider an iterative method from the combination of two inexact Newton approaches. The first one, represented by Algorithm 3.1, is globalized with a linesearch technique [11, 15], and converges slowly, for a large choice of initializations, whereas the second one, represented by Algorithm 3.2, is more standard and converges faster but for a restricted choice of initializations [4]. The GMRES algorithm [16], using a preconditioner based on an incomplete LU factorization, is incorporated into each iteration.

In Algorithms 3.1 and 3.2, the cost function $F$, defined for $\mathrm{x} \in \mathbb{R}^{N_{f}+N^{0}}$, is such that

$$
F(\mathrm{x})=\left(\begin{array}{cc}
A & B \\
C & D
\end{array}\right) \mathrm{x}+\left(\begin{array}{c}
\mathcal{N}\left(\mathrm{x}_{N_{f}}\right) \\
0
\end{array}\right)-\left(\begin{array}{l}
F_{1} \\
F_{2}
\end{array}\right) \in \mathbb{R}^{N_{f}+N^{0}}
$$

where $\mathrm{x}=\left(\mathrm{x}_{N_{f}} \mid \mathrm{x}_{N^{0}}\right)^{T}, \mathrm{x}_{N_{f}} \in \mathbb{R}^{N_{f}}, \mathrm{x}_{N^{0}} \in \mathbb{R}^{N^{0}}$. Thus, determining a solution $(\mathcal{P}, \Phi)$ of (3.2) is equivalent to find $\mathrm{x}$ satisfying $F(\mathrm{x})=0$, by considering the correspondences $\mathrm{x}_{N_{f}} \equiv \mathcal{P}$ and $\mathrm{x}_{N^{0}} \equiv \Phi$. 
$\overline{\text { Algorithm 3.1 (Inexact quasi-Newton algorithm with search for a step-length parame- }}$ ter $\alpha$ )

1. Let $\mathrm{x}:=\mathrm{x}_{0}$, where $\mathrm{x}_{0}$ is given. Evaluate $F(\mathrm{x})$. If $\|F(\mathrm{x})\|_{2} \approx 0$, stop the algorithm and return $\mathrm{x}$;

2. Let $k:=1$;

3. Evaluate $J_{F}(\mathrm{x})$, and compute an approximate solution, $\delta \mathrm{x}$, of the matrix equation $J_{F}(\mathrm{x}) \delta \mathrm{x}=-F(\mathrm{x})$, with the restarted GMRES algorithm (preconditioned by an incomplete $\mathrm{LU}$ factorization), satisfying $\left\|F(\mathrm{x})+J_{F}(\mathrm{x}) \delta \mathrm{x}\right\|_{2} \leq \eta\|F(\mathrm{x})\|_{2}$, where $\eta:=\min \left\{0.5,\|F(\mathrm{x})\|_{2}\right\}$;

4. Compute $\alpha>0$ :

4.1. Let $\alpha^{(0)}:=1$;

4.2. Evaluate $f\left(\mathrm{x}+\alpha^{(0)} \delta \mathrm{x}\right), f(\mathrm{x})$ and $\nabla f(\mathrm{x})$. If $f\left(\mathrm{x}+\alpha^{(0)} \delta \mathrm{x}\right) \leq f(\mathrm{x})+$ $\beta_{1} \alpha^{(0)} \nabla f(\mathrm{x}) \cdot \delta \mathrm{x}$ and $\nabla f\left(\mathrm{x}+\alpha^{(0)} \delta \mathrm{x}\right) \cdot \delta \mathrm{x} \geq \beta_{2} \nabla f(\mathrm{x}) \cdot \delta \mathrm{x}$, let $\alpha:=\alpha^{(0)}$ and go to Step 5;

4.3. Compute $\alpha^{(1)}:=\frac{\|F(\mathrm{x})\|_{2}^{2}\left(\alpha^{(0)}\right)^{2}}{\left\|F\left(\mathrm{x}+\alpha^{(0)} \delta \mathrm{x}\right)\right\|_{2}^{2}+\left(2 \alpha^{(0)}-1\right)\|F(\mathrm{x})\|_{2}^{2}} ;$

4.4. Evaluate $f\left(\mathrm{x}+\alpha^{(1)} \delta \mathrm{x}\right)$. If $f\left(\mathrm{x}+\alpha^{(1)} \delta \mathrm{x}\right) \leq f(\mathrm{x})+\beta_{1} \alpha^{(1)} \nabla f(\mathrm{x}) \cdot \delta \mathrm{x}$ and $\nabla f\left(\mathrm{x}+\alpha^{(1)} \delta \mathrm{x}\right) \cdot \delta \mathrm{x} \geq \beta_{2} \nabla f(\mathrm{x}) \cdot \delta \mathrm{x}$, let $\alpha:=\alpha^{(1)}$ and go to Step 5;

4.5. Let $j:=2$;

4.6. Compute $\alpha^{(j)}:=\frac{-b+\sqrt{b^{2}+3 a\|F(\mathrm{x})\|_{2}^{2}}}{3 a}$, where

$$
\begin{aligned}
\left(\begin{array}{c}
a \\
b
\end{array}\right):= & \frac{1}{\alpha^{(j-1)}-\alpha^{(j-2)}}\left(\begin{array}{cc}
\frac{1}{\left(\alpha^{(j-1)}\right)^{2}} & -\frac{1}{\left(\alpha^{(j-2)}\right)^{2}} \\
-\frac{\alpha^{(j-2)}}{\left(\alpha^{(j-1)}\right)^{2}} & \frac{\alpha^{(j-1)}}{\left(\alpha^{(j-2)}\right)^{2}}
\end{array}\right) \\
& \times\left(\begin{array}{c}
f\left(\mathrm{x}+\alpha^{(j-1)} \delta \mathrm{x}\right)-f(\mathrm{x})-(\nabla f(\mathrm{x}) \cdot \delta \mathrm{x}) \alpha^{(j-1)} \\
f\left(\mathrm{x}+\alpha^{(j-2)} \delta \mathrm{x}\right)-f(\mathrm{x})-(\nabla f(\mathrm{x}) \cdot \delta \mathrm{x}) \alpha^{(j-2)}
\end{array}\right)
\end{aligned}
$$

4.7. Evaluate $f\left(\mathrm{x}+\alpha^{(j)} \delta \mathrm{x}\right)$. If $f\left(\mathrm{x}+\alpha^{(j)} \delta \mathrm{x}\right) \leq f(\mathrm{x})+\beta_{1} \alpha^{(j)} \nabla f(\mathrm{x}) \cdot \delta \mathrm{x}$ and $\nabla f\left(\mathrm{x}+\alpha^{(j)} \delta \mathrm{x}\right) \cdot \delta \mathrm{x} \geq \beta_{2} \nabla f(\mathrm{x}) \cdot \delta \mathrm{x}$, let $\alpha:=\alpha^{(j)}$ and go to Step 5;

4.8. Update $j:=j+1$ and return to Substep 4.6;

5. Update $\mathrm{x}:=\mathrm{x}+\alpha \delta \mathrm{x}$;

6. Update $k:=k+1$. If $k$ is too large, stop the algorithm and return $\mathrm{x}$;

7. Evaluate $F(\mathrm{x})$. If $\|F(\mathrm{x})\|_{2} \approx 0$, and $\frac{\|F(\mathrm{x})\|_{2}}{\left\|F\left(\mathrm{x}_{0}\right)\right\|_{2}} \approx 0$, stop the algorithm and return $\mathrm{x}$;

8. Return to Step 3. 
Algorithm 3.2 (Inexact Newton algorithm)

1. Let $\mathrm{x}:=\mathrm{x}_{0}$, where $\mathrm{x}_{0}$ is given. Evaluate $F(\mathrm{x})$. If $\|F(\mathrm{x})\|_{2} \approx 0$, stop the algorithm and return $\mathrm{x}$;

2. Let $k:=1$;

3. Evaluate $J_{F}(\mathrm{x})$, and compute an approximate solution, $\delta \mathrm{x}$, of the matrix equation $J_{F}(\mathrm{x}) \delta \mathrm{x}=-F(\mathrm{x})$, with the restarted GMRES algorithm (preconditioned by an incomplete LU factorization), satisfying $\left\|F(\mathrm{x})+J_{F}(\mathrm{x}) \delta \mathrm{x}\right\|_{2} \leq \eta\|F(\mathrm{x})\|_{2}$, where $\eta:=\min \left\{0.5,\|F(\mathrm{x})\|_{2}\right\}$;

4. Update $\mathrm{x}:=\mathrm{x}+\delta \mathrm{x}$;

5. Update $k:=k+1$. If $k$ is too large, stop the algorithm and return $\mathrm{x}$;

6. Evaluate $F(\mathrm{x})$. If $\|F(\mathrm{x})\|_{2} \approx 0$, and $\frac{\|F(\mathrm{x})\|_{2}}{\left\|F\left(\mathrm{x}_{0}\right)\right\|_{2}} \approx 0$, stop the algorithm and return $\mathrm{x}$;

7. Return to Step 3.

Algorithm 3.2 is based on an inexact Newton technique that consists of finding a sequence $\left(\mathrm{x}_{k}\right)_{k \geq 0}$ converging to a zero of $F$. In the standard Newton method, at each iteration $k$, the vector $\mathrm{x}_{k+1}$ is equal to $\mathrm{x}_{k}+\delta \mathrm{x}_{k}$, where $\delta \mathrm{x}_{k}$ is the solution of the matrix equation $J_{F}\left(\mathrm{x}_{k}\right) \delta \mathrm{x}_{k}=-F\left(\mathrm{x}_{k}\right)$, of course under the requirement that $J_{F}\left(\mathrm{x}_{k}\right)$, the jacobian matrix related to $F$ and evaluated at $\mathrm{x}_{k}$, is invertible. Here, by representing by $\|\cdot\|_{2}$ the euclidian norm on $\mathbb{R}^{N_{f}+N^{0}}$, we consider rather an approximation of $\delta \mathrm{x}_{k}$ subject to the relation below

$$
\left\|F\left(\mathrm{x}_{k}\right)+J_{F}\left(\mathrm{x}_{k}\right) \delta \mathrm{x}_{k}\right\|_{2} \leq \eta_{k}\left\|F\left(\mathrm{x}_{k}\right)\right\|_{2}
$$

with $\eta_{k}=\min \left\{0.5,\left\|F\left(\mathrm{x}_{k}\right)\right\|_{2}\right\}$, ensuring the convergence of the algorithm [4].

In Algorithm 3.1, we deal with the function $f$ defined for $\mathrm{x} \in \mathbb{R}^{N_{f}+N^{0}}$ as follows:

$$
f(\mathrm{x})=\frac{1}{2}\|F(\mathrm{x})\|_{2}^{2} \in \mathbb{R} .
$$

Every solution $\mathrm{x}, \mathrm{x} \in \mathbb{R}^{N_{f}+N^{0}}$, of the equation $F(\mathrm{x})=0$ satisfies

$$
f(\mathrm{x})=\min \left\{f(\mathrm{y}) ; \mathrm{y} \in \mathbb{R}^{N_{f}+N^{0}}\right\} .
$$

We use a linesearch technique, which consists of finding a sequence $\left(\mathrm{x}_{k}\right)_{k \geq 0}$ converging to a minimum of $f$, with the help of a sequence of step lengths $\left(\alpha_{k}\right)_{k \geq 0}$ such that, at each iteration $k, f\left(\mathrm{x}_{k}+\alpha_{k} \delta \mathrm{x}_{k}\right)$ is "sufficiently smaller" than $f\left(\mathrm{x}_{k}\right)$, where $\delta \mathrm{x}_{k}$ satisfies (3.3). More precisely, we assume that the Wolfe conditions [21] are satisfied

$$
\begin{aligned}
& f\left(\mathrm{x}_{k}+\alpha_{k} \delta \mathrm{x}_{k}\right) \leq f\left(\mathrm{x}_{k}\right)+\beta_{1} \alpha_{k} \nabla f\left(\mathrm{x}_{k}\right) \cdot \delta \mathrm{x}_{k}, \\
& \nabla f\left(\mathrm{x}_{k}+\alpha_{k} \delta \mathrm{x}_{k}\right) \cdot \delta \mathrm{x}_{k} \geq \beta_{2} \nabla f\left(\mathrm{x}_{k}\right) \cdot \delta \mathrm{x}_{k}
\end{aligned}
$$


with $0<\beta_{1}<\beta_{2}<1$, ensuring the convergence of the algorithm [15]. In order to find such a suitable coefficient $\alpha_{k}$, we deal with a backtracking method [15]. Namely, we use a sequence $\left(\alpha_{k}^{(j)}\right)_{j \geq 0}$ converging to a minimum of the function $\theta_{k}$, defined for each $\alpha \in \mathbb{R}$ as

$$
\theta_{k}(\alpha)=f\left(\mathrm{x}_{k}+\alpha \delta \mathrm{x}_{k}\right) \in \mathbb{R} .
$$

We consider $\alpha_{k}$ as being the first term $\alpha_{k}^{\left(j_{\star}\right)}$ of the sequence $\left(\alpha_{k}^{(j)}\right)_{j \geq 0}$ such that the relations in (3.4) are satisfied by substituting therein the coefficient $\alpha_{k}$ for $\alpha_{k}^{\left(j_{\star}\right)}$. More precisely, for building such a sequence, let us set $\alpha_{k}^{(0)}>0$, by considering for instance $\alpha_{k}^{(0)}:=1$. We compute $\alpha_{k}^{(1)}$ as being the minimum of a quadratic interpolation $\theta_{k}^{\text {quad }}$ of $\theta_{k}$, defined for each $\alpha \in \mathbb{R}$ as

$$
\theta_{k}^{\text {quad }}(\alpha)=\frac{\alpha^{2}}{\left(\alpha_{k}^{(0)}\right)^{2}}\left(\theta_{k}\left(\alpha_{k}^{(0)}\right)-\theta_{k}(0)-\alpha_{k}^{(0)} \theta_{k}^{\prime}(0)\right)+\theta_{k}^{\prime}(0) \alpha+\theta_{k}(0) .
$$

Thus,

$$
\begin{aligned}
\alpha_{k}^{(1)} & =-\frac{\theta_{k}^{\prime}(0)\left(\alpha_{k}^{(0)}\right)^{2}}{2\left(\theta_{k}\left(\alpha_{k}^{(0)}\right)-\theta_{k}(0)-\theta_{k}^{\prime}(0) \alpha_{k}^{(0)}\right)} \\
& =\frac{\left\|F\left(\mathrm{x}_{k}\right)\right\|_{2}^{2}\left(\alpha_{k}^{(0)}\right)^{2}}{\left\|F\left(\mathrm{x}_{k}+\alpha_{k}^{(0)} \delta \mathrm{x}_{k}\right)\right\|_{2}^{2}+\left(2 \alpha_{k}^{(0)}-1\right)\left\|F\left(\mathrm{x}_{k}\right)\right\|_{2}^{2}}
\end{aligned}
$$

For $j \geq 2, \alpha_{k}^{(j)}$ is chosen as being the (local) minimum of a cubic approximation $\theta_{k}^{\text {cub }}$ of $\theta_{k}$, expressed for each $\alpha \in \mathbb{R}$ as below

$$
\theta_{k}^{\mathrm{cub}}(\alpha)=a \alpha^{3}+b \alpha^{2}+\theta_{k}^{\prime}(0) \alpha+\theta_{k}(0),
$$

where

$$
\begin{aligned}
\left(\begin{array}{c}
a \\
b
\end{array}\right)= & \frac{1}{\alpha_{k}^{(j-1)}-\alpha_{k}^{(j-2)}}\left(\begin{array}{cc}
\frac{1}{\left(\alpha_{k}^{(j-1)}\right)^{2}} & -\frac{1}{\left(\alpha_{k}^{(j-2)}\right)^{2}} \\
-\frac{\alpha_{k}^{(j-2)}}{\left(\alpha_{k}^{(j-1)}\right)^{2}} & \frac{\alpha_{k}^{(j-1)}}{\left(\alpha_{k}^{(j-2)}\right)^{2}}
\end{array}\right) \\
& \times\left(\begin{array}{c}
\theta_{k}\left(\alpha_{k}^{(j-1)}\right)-\theta_{k}(0)-\theta_{k}^{\prime}(0) \alpha_{k}^{(j-1)} \\
\theta_{k}\left(\alpha_{k}^{(j-2)}\right)-\theta_{k}(0)-\theta_{k}^{\prime}(0) \alpha_{k}^{(j-2)}
\end{array}\right) .
\end{aligned}
$$

We consider thus, for $j \geq 2$

$$
\alpha_{k}^{(j)}=\frac{-b+\sqrt{b^{2}-3 a \theta_{k}^{\prime}(0)}}{3 a}=\frac{-b+\sqrt{b^{2}+3 a\left\|F\left(\mathrm{x}_{k}\right)\right\|_{2}^{2}}}{3 a} .
$$


Algorithm 3.3 (Main algorithm)

1. Choose an initial datum $\mathrm{x}_{0} \in \mathbb{R}^{N_{f}+N^{0}}$;

2. Call Algorithm 3.1;

3. Update $\mathrm{x}_{0}:=\mathrm{x}$, where $\mathrm{x}$ is the output of Algorithm 3.1;

4. Call Algorithm 3.2.

Algorithm 3.3 is performed for determining solutions of (3.2). It requires Algorithm 3.1 as well as Algorithm 3.2, where we make in the implementation stage the choice to evaluate numerically $J_{F}$ and $\nabla f$ at each point from their formal expressions, as in [11]. An efficiency of this main algorithm was numerically observed in [11] from investigations in a context not dedicated to applications in particular; namely, with the considerations $\beta_{1}=10^{-4}$ and $\beta_{2}=10^{-1}$ that were suggested from [15].

Of course, by dealing with a framework where the expressions of theoretical solutions are available, even without being related to a specific application, our numerical inspections underline quantitatively the efficiency of this main algorithm.

As an example of this framework, let us consider that $\Omega$ and $\Omega_{f}$ are polyhedral, and shaped like two cylinders sharing the same center, namely the origin, and the same axis which is $(O z)$. The radii of the "cylinders" represented by $\Omega$ and $\Omega_{f}$ are fixed as equal to 1.2 and 0.4 , respectively. Moreover, the heights of these "cylinders" are fixed as equal to 1.7 and 0.1 , respectively. Also, let us consider

$$
\begin{array}{ll}
\varkappa_{\|}=10, \quad P_{0}=1, & \xi=\left(\xi_{i, j}\right)_{1 \leq i, j \leq 3}, \\
\left.\varepsilon\right|_{\Omega_{f}}=\left(\varepsilon_{i, j}^{f}\right)_{1 \leq i, j \leq 3}, & \left.\varepsilon\right|_{\Omega_{p}}=\left(\varepsilon_{i, j}^{p}\right)_{1 \leq i, j \leq 3},
\end{array}
$$

where $\xi_{i, j}=\varepsilon_{i, j}^{f}=\varepsilon_{i, j}^{p}=0$ for $1 \leq i \neq j \leq 3$. The other coefficients of these tensors are

$$
\begin{array}{ll}
\xi_{1,1}=\xi_{2,2}=\xi_{3,3}=0.1, & \varepsilon_{1,1}^{f}=\varepsilon_{2,2}^{f}=10, \\
\varepsilon_{1,1}^{p}=\varepsilon_{2,2}^{p}=\varepsilon_{3,3}^{p}=\varepsilon_{p}>0, & \varepsilon_{3,3}^{f}=\varepsilon_{\| z}>0 .
\end{array}
$$

Let us set $\varphi_{S}(x, y, z)=x y$ for $(x, y, z)^{T} \in S$. Although by fixing $\varphi_{S}$ in this way, it does not refer to a concrete consideration with regard to the voltage potential applications, a theoretical solution of (EGL) can be expressed. It follows in particular, when $t=-1$, that $(P, \varphi)$, where $P(x, y, z)=0$ for $(x, y, z)^{T} \in \Omega_{f}, \varphi(x, y, z)=x y$ for $(x, y, z)^{T} \in \Omega$, satisfies (EGL). Correlatively, $(P, \phi)$ is subject to (2.12) and (2.13), with $\phi=\varphi-w$, and $w$ taken as in Theorem 2.1. Let us fix $\varepsilon_{\| z}=0.1, \varepsilon_{p}=10$, and consider three meshes of $\bar{\Omega}$ achieved in accordance with Subsection 3.1. The first mesh involves 25183 degrees of freedom and is associated with $h=h_{1}=1.6610^{-1}$ (which becomes equal to $7.3110^{-2}$ under restriction to $\overline{\Omega_{f}}$ ). The second mesh deals with 193675 degrees of freedom and is associated with $h=h_{2}=8.7110^{-2}$ (which becomes equal to $4.4410^{-2}$ under restriction 
to $\overline{\Omega_{f}}$ ). The third mesh involves 1499982 degrees of freedom and is associated with $h=h_{3}=4.7910^{-2}$ (which becomes equal to $2.2810^{-2}$ under restriction to $\overline{\Omega_{f}}$ ).

Let us denoted by $\left\{\mathrm{x}_{k}^{(i)}\right\}_{1 \leq k \leq N^{0(i)}}$ the set of internal vertices of the $i$-th mesh of $\bar{\Omega}$, and consider

$$
\|u\|_{2}^{(i)}=\left(\frac{1}{N^{0(i)}} \sum_{k=1}^{N^{0(i)}}\left|u\left(\mathrm{x}_{k}^{(i)}\right)\right|^{2}\right)^{\frac{1}{2}},
$$

for $u: \bar{\Omega} \longrightarrow \mathbb{R}$, where $i$ is fixed, $1 \leq i \leq 3$. Also, for the discrete solution $\left(P^{(i)}, \varphi^{(i)}\right)$ associated with $(P, \varphi)$ and obtained from the $i$-th mesh, let us denote by

$$
\operatorname{err}_{2}^{(i)}=\frac{\left\|\varphi-\varphi^{(i)}\right\|_{2}^{(i)}}{\|\varphi\|_{2}^{(i)}}
$$

the error evaluated relatively to $\varphi$.

From an initialization of the main algorithm with $(1, \ldots, 1)^{T} \in \mathbb{R}^{N^{0(i)}}$, after taking $w$ in accordance of course with Theorem 2.1 and here as a polynomial of degree equal to 2 , we obtain that the values corresponding to $\operatorname{err}_{2}^{(i)}$ are very negligible, namely, $\operatorname{err}_{2}^{(1)}=$ $3.6110^{-6} \%$, err $_{2}^{(2)}=7.6210^{-7 \%}$, err ${ }_{2}^{(3)}=1.3310^{-7 \%}$. The same observation arises by considering several other choices for the initialization of this algorithm. It results moreover that the values corresponding to the absolute errors about $P$, as regards then $\overline{\Omega_{f}}$, are also negligible.

Another example of the mentioned framework is the one where we consider $t=-2$, with $\varkappa_{\|} \neq 0, \varepsilon_{\| z} \neq \varepsilon_{p}$ again, by keeping the previous data for the other parameters. Although by assigning such a value to $t$, which is not then related to a concrete consideration with regard to the temperature applications, a theoretical solution of (EGL) can be expressed; namely, when we set

$$
\varphi_{S}(x, y, z)=\frac{4 \pi}{\varepsilon_{\| z}-\varepsilon_{p}} z, \quad(x, y, z)^{T} \in S .
$$

It follows in particular with $\varkappa_{\|}=1$ that $(P, \varphi)$, where

$$
\begin{array}{lll}
P(x, y, z) & =1, & (x, y, z)^{T} \in \Omega_{f}, \\
\varphi(x, y, z)=\frac{4 \pi}{\varepsilon_{\| z}-\varepsilon_{p}} z, & (x, y, z)^{T} \in \Omega,
\end{array}
$$

satisfies (EGL). Correlatively, $(P, \phi)$ is subject to (2.12) and (2.13), with $\phi=\varphi-w$, and $w$ taken as in Theorem 2.1.

By fixing $\varepsilon_{\| z}=2, \varepsilon_{p}=1$, and considering the previous meshes of $\bar{\Omega}$, as well as $(0, \ldots, 0)^{T} \in \mathbb{R}^{N^{0(i)}}$ for the initialization of the algorithm, with $w$ here as a polynomial of degree equal to 1 , we obtain that the values corresponding to $\operatorname{err}_{2}^{(i)}$ are very 
negligible too. The same observation derives from several other choices for the initialization of this algorithm. Moreover, it arises that the values corresponding to the errors determined similarly and relatively to $P$, as regards then $\overline{\Omega_{f}}$, are also negligible here.

In addition to the efficiency of this algorithm on the quantitative aspect, let us mention that our numerical inspections underline an associated approach which provides very satisfactory results from a qualitative point of view. Namely, with respect to (small) values of the parameter $h$, the same types of transition jumps of solutions are numerically provided for a same set of data associated with physical and geometrical parameters. Let us fix $\varkappa_{\|}=10, t=-20, \varepsilon_{\| z}=0.1, \varepsilon_{p}=0.1$, by keeping the previous values for the other parameters, and consider the three previous meshes of $\bar{\Omega}$, where of course $h_{j}<h_{i}$, for $1 \leq i<j \leq 3$. As previously, this arbitrary value assigned to $t$ is however unrelated to a concrete consideration with regard to the temperature applications. With $\phi_{0} \in \mathbb{R}$, let us set $\varphi_{S}(x, y, z)=-\frac{2 z}{H} \phi_{0}$ for $(x, y, z)^{T} \in S$, by indicating that $H=1.7$ is the height of the "cylinder" represented by $\Omega$.

In contrast to the previous cases, the expressions of theoretical solutions are not here available.

In addition to the above notation, let us consider $\|u\|_{\infty}^{(i)}=\max _{1 \leq k \leq N^{0(i)}}\left|u\left(\mathrm{x}_{k}^{(i)}\right)\right|$, for $u: \bar{\Omega} \longrightarrow \mathbb{R}$, where $\left\{\mathrm{x}_{k}^{(i)}\right\}_{1 \leq k \leq N^{0(i)}}$ corresponds again to the set of internal vertices of the $i$-th mesh of $\bar{\Omega}$, with $i$ fixed, $1 \leq i \leq 3$. For a discrete solution $\left(P^{(i)}, \varphi^{(i)}\right)$ obtained from the $i$-th mesh and a discrete solution $\left(P^{(j)}, \varphi^{(j)}\right)$ resulting from the $j$-th mesh, which are associated with (EGL) and where $1 \leq i<j \leq 3$, we deal with the error between $\varphi^{(j)}$ and $\varphi^{(i)}$, evaluated relatively to $\varphi^{(j)}$. Let us then set

$$
e r r_{2}^{(i, j)}=\frac{\left\|\varphi^{(j)}-\varphi^{(i)}\right\|_{2}^{(j)}}{\left\|\varphi^{(j)}\right\|_{2}^{(j)}}, \quad \operatorname{err} r_{\infty}^{(i, j)}=\frac{\left\|\varphi^{(j)}-\varphi^{(i)}\right\|_{\infty}^{(j)}}{\left\|\varphi^{(j)}\right\|_{\infty}^{(j)}}
$$

The results represented in Fig. 1 are related to the consideration $U=2 \phi_{0}$, from the choice of $\varphi_{S}$ (see also [11]). The function $w$, taken as in Theorem 2.1, is in particular

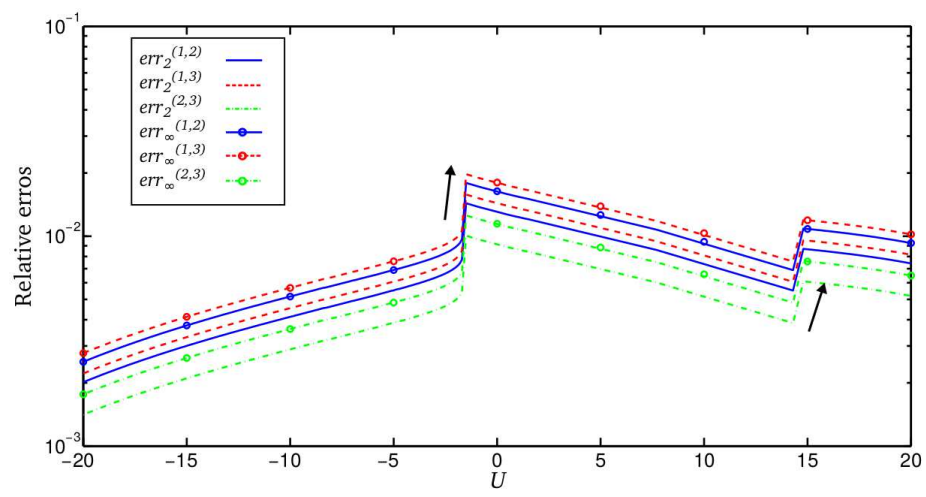

Figure 1: Semi-log representation of $\operatorname{err}_{2}^{(i, j)}, \operatorname{err}_{\infty}^{(i, j)}(1 \leq i<j \leq 3)$ with respect to $U=2 \phi_{0}$. 
here a polynomial of degree equal to 1 . Various values are taken for $\phi_{0}$; this refers to a variation of $\varphi_{S}$. These results concern the numerical values (not expressed in percent) that are obtained for $\operatorname{err}_{2}^{(i, j)}, \operatorname{err}_{\infty}^{(i, j)}$, from the three previous meshes.

The solutions arising from these meshes have the same profiles. Moreover, the transition jumps of solutions are associated with the same values of $U$. Also, the maximal value of all the errors $\operatorname{err}_{2}^{(i, j)}$, $\operatorname{err}_{\infty}^{(i, j)}$, for $1 \leq i<j \leq 3$, remains of course small. Similar results derive from the evaluations of errors about $P^{(i)}$. Namely, smaller values are obtained in the determination of these errors associated with $\overline{\Omega_{f}}$.

\section{Numerical experiments}

We are interested here in particular in developments which aim at impregnating physical experiments. After describing the computational configurations, we investigate the state switchings related to (2.1)-(2.7) as well as calibration effects based on the numerical variations of parameters of this model.

\subsection{Computational configurations}

We distinguish two types of computational configurations. The first one is devoted to cylindrical geometries while the second one is related to parallelepipedic geometries. For the first type, $\Omega_{f}$ and $\Omega$ are polyhedral and shaped like cylinders; they share the same center, namely the origin, and the same axis, represented by $(O z)$. We denote by $R_{f}$ the radius and $H_{f}$ the height of the "cylinder" represented by $\Omega_{f}$. In a similar way, the radius and the height of the "cylinder" represented by $\Omega$ are denoted by $R$ and $H$, respectively. For the second type, $\Omega_{f}$ and $\Omega$ are parallelepipedic. The mesh of $\bar{\Omega}$ associated with each configuration is achieved in accordance with the previous section. Namely, we consider two reference configurations, dealing with volumes approximately equal as well as with the same height as regards $\Omega_{f}$, and in association with the meshes represented by:

- $\mathcal{T}_{h}^{(1)}$, in the cylindrical frame with $\left(R_{f}, H_{f}\right)=(8,1.7)$ and $(R, H)=(12,17)$;

- $\mathcal{T}_{h}^{(2)}$, in the parallelepipedic context, where

$$
\overline{\Omega_{f}}=\left[-R_{f}, R_{f}\right] \times\left[-R_{f}, R_{f}\right] \times\left[-\frac{H_{f}}{2}, \frac{H_{f}}{2}\right]
$$

with thus $\left(R_{f}, H_{f}\right)=(7.09,1.7)$, and

$$
\bar{\Omega}=[-R, R] \times[-R, R] \times\left[-\frac{H}{2}, \frac{H}{2}\right]
$$

with $(R, H)=(15,30)$. 
Let us already mention that several values, for the mesh size $h$, have been used in numerical experiments, relatively to each configuration. In the next subsections, the results that will be presented derive, in the absence of any other explicit mention, from experiments with the consideration $h=0.61$, involving 485621 degrees of freedom for $\mathcal{T}_{h}^{(1)}$ and 1121858 in the frame of $\mathcal{T}_{h}^{(2)}$. Correlatively, this mesh size becomes equal to 0.47 under restriction to $\overline{\Omega_{f}}$.

Regarding the physical parameters, we consider for each computational configuration, by denoting by $I_{3}$ the $3 \times 3$ identity tensor

$$
\left.\varepsilon\right|_{\Omega_{f}}=\left(\begin{array}{ccc}
10 & 0 & 0 \\
0 & 5 & 0 \\
0 & 0 & 1
\end{array}\right),\left.\quad \varepsilon\right|_{\Omega_{p}}=\varepsilon_{p} I_{3},
$$

$\varepsilon_{p}=90, \xi=I_{3}, P_{0}=1, \varkappa_{\|}=11.5$. Let us already indicate, in particular, that variations of the parameters $R_{f}, H_{f}, t$ and $\varepsilon_{p}$ will be performed in Subsection 4.3.

With $\phi_{0} \in \mathbb{R}$, we define $\varphi_{S}$ as

$$
(x, y, z) \in S \longmapsto \varphi_{S}(x, y, z)=\frac{H-2 z}{H} \phi_{0}-\phi_{0} \in \mathbb{R}
$$

in such a way that $\varphi_{S}\left(x, y,-\frac{H}{2}\right)=\phi_{0}$ and $\varphi_{S}\left(x, y, \frac{H}{2}\right)=-\phi_{0}$. This is in association with the voltage potential $U$ applied to the device

$$
U=2 \phi_{0}
$$

as well as with the consideration of $w$ in (3.1), as in (2.12) and (2.13), such that

$$
w(x, y, z)=\frac{H-2 z}{H} \phi_{0}-\phi_{0}
$$

for $(x, y, z)^{T} \in \Omega$. Namely, $\left.w\right|_{S}=\varphi_{S}$.

In the next subsections, we will describe the numerical results, in each configuration, through also the behavior of the discrete "average polarization". This scalar field is represented by $\overline{P_{h}}$,

$$
\overline{P_{h}}=\frac{1}{\left|\Omega_{f}\right|} \int_{\Omega_{f}} P_{h} \mathrm{dx}
$$

and arises from the finite element approximation of a state $(P, \varphi)$ subject of course to (EGL).

Although in the applications the reduced temperature $t$ takes values in $[-1,0)$, as reported in Section 2, we will be interested in the extended consideration where $t \in$ $[-1,0]$.

\subsection{Numerical study of state switchings}

We make here use of a protocol presented in $[11,12]$ which is suitable for the determination of non obvious states and incorporates also a process of "heating" as 
well as of "cooling" of a ferroelectric device. We initialize Algorithm 3.3 with random vectors of size $N_{f}+N^{0}$, distributed according to the uniform law of $[-1,1]^{N_{f}+N^{0}}$, as well as with deterministic vectors.

We obtain several profiles; each one allowing us to identify the (discrete) states $\left(P_{h}, \varphi_{h}\right)$, namely subject to (EGL), and for which the polarization texture is similar. Fig. 2 presents some of these profiles, resulting from $\mathcal{T}_{h}^{(1)}$ and $\mathcal{T}_{h}^{(2)}$, with $t=-0.9$, $U=0$. They are distinguished by the shape and by the number of polarization domains as being:

- Monodomain Profile (MP), case of a unique domain of polarization (see Figs. 2(a) and 2(b));

- Concentric-cylindrical Profile (CP), context of a central domain shaped like a cylinder or distorted cylinder, surrounded by another domain (see Figs. 2(c) and $2(d))$;

- 2-Bands Profile (2BP), case of two domains, organized into bands (see Figs. 2(e) and 2(f)).

In addition to these and in particular, the Obvious Profile, denoted by (OP) in the sequel, in correspondence to the case where $P_{h} \equiv 0$, arises from simulations performed here, as well as the profile with four domains having a "diamond" shape (see Fig. 3). Note that some of the profiles obtained have already been observed in $[13,19]$, including the 2-Bands Profile experimentally found in the case of a nanofilm of Lead Titanate [19].

Each non obvious state of the device can be numerically submitted to a "heating" or a "cooling" process. Namely, in order to compute a solution of (3.2) at the temperature $t+\delta t$, where $\delta t \in \mathbb{R}^{\star}$, we initialize Algorithm 3.3 with the help of a solution resulting at the temperature $t$ (see also [11] for more details).

Fig. 3 presents the results obtained from $\mathcal{T}_{h}^{(2)}$, with respect to the temperature, when we "heat up" (with $\delta t=10^{-3}$ ) or "cool down" (with $\delta t=-10^{-3}$ ) several states, for $U=0$. While $\overline{P_{h}}$ depends continuously on the temperature, the distributions of the polarization stay similar. In this study, a discontinuity corresponds to a change of profile. For instance, (MP) exists for a range of small values taken by the reduced temperature and jumps, under heating, to (CP) at $t=-0.805$. Such a transition is qualified as irreversible since, by lowering the temperature from the new profile (here (CP)), we do not find the first profile (namely (MP)). The next jumps, thus initiated by (MP), are schematized as bellow

$$
(\mathrm{MP}) \underset{t=-0.805}{\longrightarrow}(\mathrm{CP}) \underset{t=-0.335}{\longrightarrow}(2 \mathrm{BP}) \underset{t=-0.307}{\longrightarrow}(\mathrm{OP})
$$

Here and in the next subsections, each jump corresponds to an irreversible transition. Each profile persists below a maximal temperature, beyond which it disappears; this is called the critical temperature of the profile. In this study, the profile having the highest 


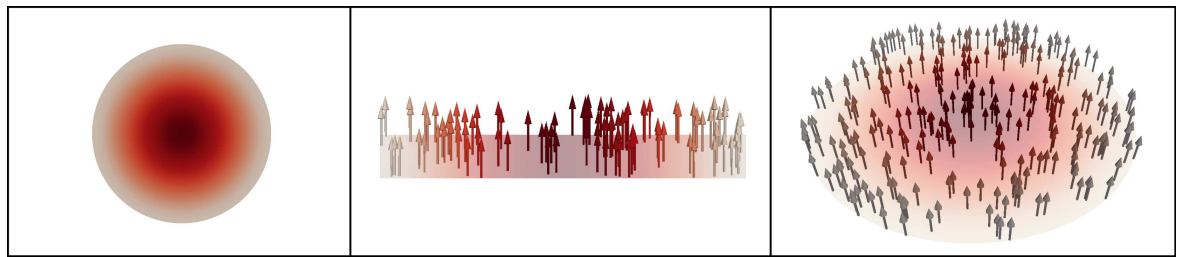

(a)-(MP)

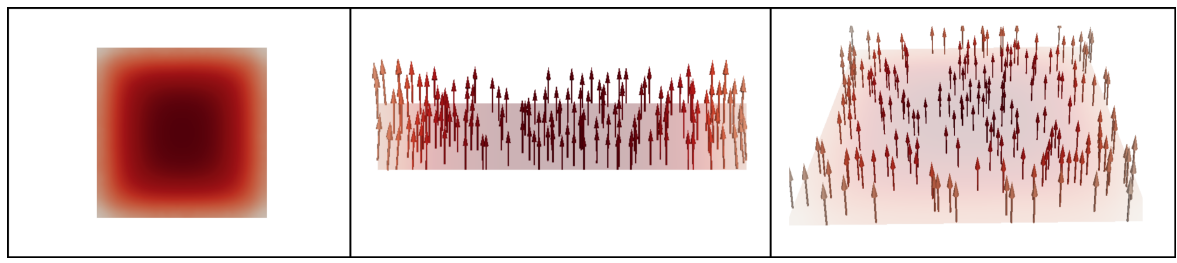

(b)-(MP)

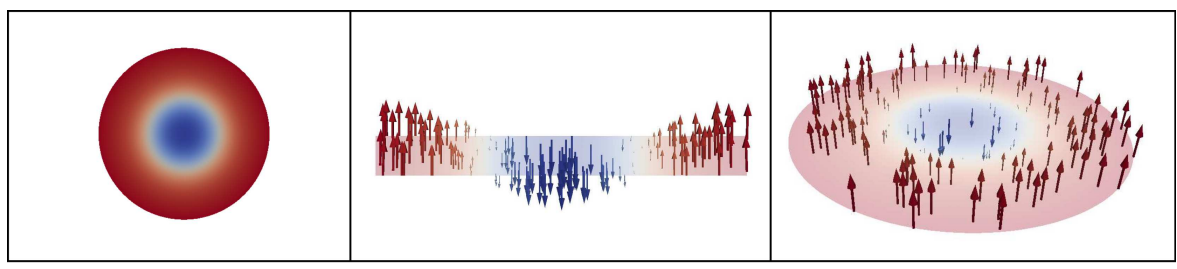

(c)-(CP)

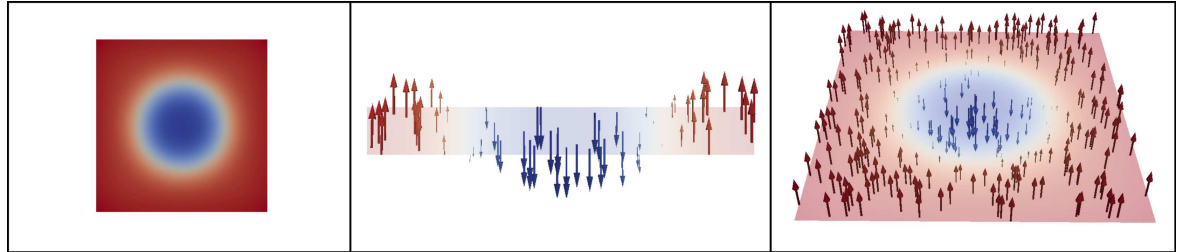

(d)-(CP)

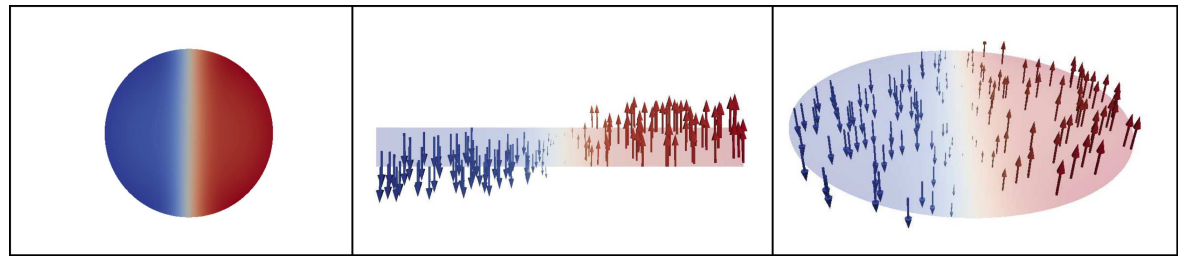

(e)-(2BP)

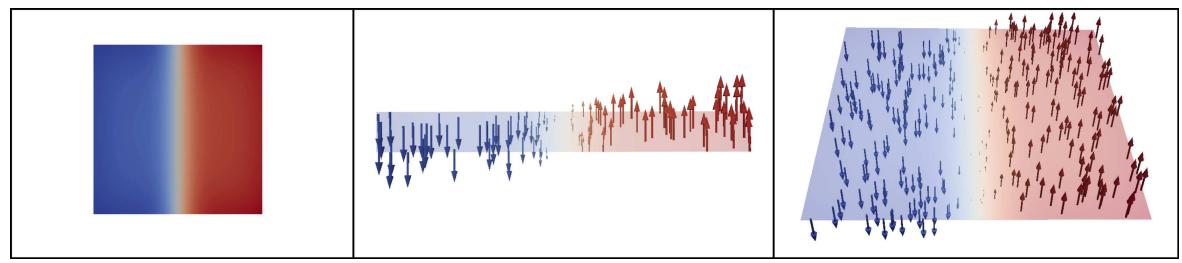

(f)-(2BP)

Figure 2: Some profiles obtained from $\mathcal{T}_{h}^{(1)}((\mathrm{a}),(\mathrm{c}),(\mathrm{e}))$ and $\mathcal{T}_{h}^{(2)}((\mathrm{b}),(\mathrm{d}),(\mathrm{f}))$, with $t=-0.9, U=0$. At the left, a cross-section of $P_{h}$ following $(z=0)$ is represented, whereas at middle and at the right, the cross-sections of the discrete electric polarization, associated with $\mathbf{P}$, following $(y=0)$ and $(z=0)$ are respectively represented. 


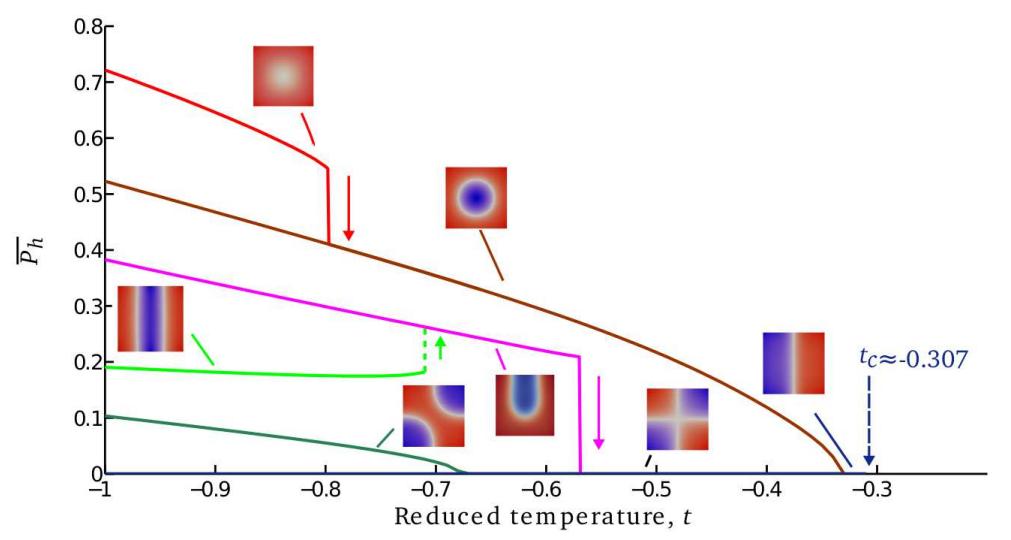

Figure 3: Representation of $\overline{P_{h}}$ with respect to the reduced temperature, from $\mathcal{T}_{h}^{(2)}$.

critical temperature, $t_{c}$, is (2BP); namely, $t_{c} \approx-0.307$. Thus, $t_{c}$ corresponds to the critical temperature of the material system.

This behavior, characteristic of ferroelectric devices [14], appears also in the context of simulations from $\mathcal{T}_{h}^{(1)}$. In particular, these same four profiles persist in this context with similar temperature ranges and transitions; the most enduring profile with nonzero polarization is also the one corresponding to (2BP). Each of the states associated with (2BP) has a constant "average polarization"; namely, $\overline{P_{h}} \equiv 0$.

Let us specify that the volume of the ferroelectric layer must be sufficiently small when compared to the one of $\Omega$, in order to avoid to disturb the emergent fringing field (see, e.g., [13] and the references therein). Of course, as long as the volume of the ferroelectric layer does not appear however as too small, numerical instabilities will not be involved; although the asymptotic framework developed by Ammari et al. [1] may remedy the contrary situation. We already mention that we deal with a ferroelectric layer for which the volume appears numerically as sufficiently small when compared to the volume of $\Omega$, but not too small, in each of the two reference configurations considered here. For instance in the context of $\mathcal{T}_{h}^{(2)}$ and as for an inspection, let us embed successively the associated ferroelectric layer in two larger paraelectric environments, by defining

$$
\begin{aligned}
& \Omega^{(3)} \equiv \bar{\Omega}=[-15,15] \times[-15,15] \times[-15,15], \\
& \Omega^{(2)}=[-16,16] \times[-16,16] \times[-16,16], \\
& \Omega^{(1)}=[-17,17] \times[-17,17] \times[-17,17] .
\end{aligned}
$$

Let us consider three meshes corresponding to triangulations of $\Omega^{(3)}, \Omega^{(2)}$ and $\Omega^{(1)}$ respectively, and having similar sizes approximately equal to 0.61 , with correlatively, as regards the ferroelectric layer, similar mesh sizes approximately equal to 0.47 . These meshes, built under the requirements of Subsection 3.1, involve respectively 1121858 , 1772141 and 2292108 degrees of freedom. Of course, a unique ferroelectric/paraelec- 
tric interface is here represented. Let us denote by $\left\{\mathrm{x}_{k}^{(i)}\right\}_{1 \leq k \leq N^{0(i)}}$ the set of internal vertices of the mesh of $\Omega^{(i)}$, where $i$ is fixed, $1 \leq i \leq 3$. For $u: \Omega^{(i)} \longrightarrow \mathbb{R}$ and $1 \leq i \leq 3$, let

$$
\|u\|_{2}^{(i)}=\left(\frac{1}{N^{0(i)}} \sum_{k=1}^{N^{0(i)}}\left|u\left(\mathrm{x}_{k}^{(i)}\right)\right|^{2}\right)^{\frac{1}{2}} .
$$

For a finite element approximation $\left(P^{(i)}, \varphi^{(i)}\right)$ obtained from $\Omega^{(i)}$ and associated with a state subject to (EGL) with $\varphi_{S}=0$, we consider the following error

$$
e r r_{2}^{(i)}=\frac{\left\|\varphi^{(1)}-\varphi^{(i)}\right\|_{2}^{(i)}}{\left\|\varphi^{(1)}\right\|_{2}^{(i)}}
$$

evaluated relatively to $\varphi^{(1)}$. By inspecting the behavior of the profile (MP), with respect to the reduced temperature, from $t=-1$ and with $\delta t=510^{-3}$, it follows, based on $\Omega^{(i)}(i=2,3)$, that the jump to (CP) occurs again, namely at $t=-0.80$. As it can be observed for instance from the results represented in Fig. 4, the errors $\operatorname{err}_{2}^{(i)}(i=2,3)$ associated with the profile (MP), or (CP), are less than $1 \%$. The similar evaluations of errors about $P^{(i)}$ provide values which remain also smaller than $1 \%$. The extension of the present paraelectric environment related to $\bar{\Omega}$ appears then numerically as without particular significant influence. Let us indicate that a similar study has been done, by obtaining relative errors now less than $6 \%$, in the context of $\mathcal{T}_{h}^{(1)}$. Also, let us mention that the CPU time corresponding to one simulation (computation of $\left(P^{(i)}, \varphi^{(i)}\right)$ based on $\Omega^{(i)}, 1 \leq i \leq 3$ ) is approximately equal to $2 \mathrm{~h}, 3 \mathrm{~h} 30$ or $8 \mathrm{~h}$ on an "Intel Xeon Processor E5520 with a frequency of $2.27 \mathrm{GHz}$ ", in the frame of $\Omega^{(3)}, \Omega^{(2)}$ or $\Omega^{(1)}$ respectively.

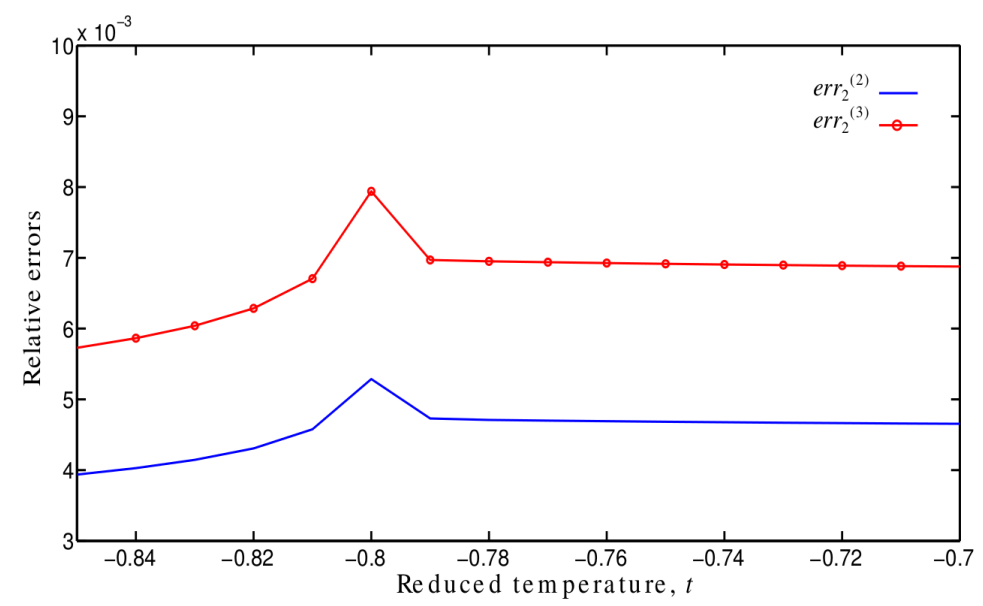

Figure 4: Representation of the relative errors $\operatorname{err}_{2}^{(2)}$ and $\operatorname{err}_{2}^{(3)}$ with respect to the reduced temperature, from parallelepipedic configurations with extensions of the paraelectric environment and vanishing boundary voltage potentials. 


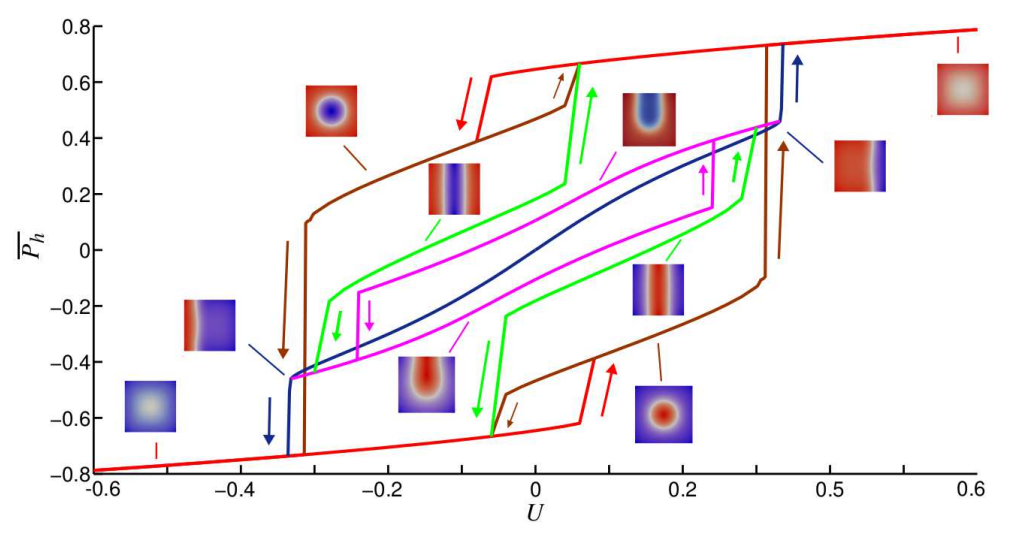

Figure 5: Hysteresis loops, for $t=-0.9$, from $\mathcal{T}_{h}^{(2)}$.

We adopt for the numerical study of the existence of hysteresis loops a protocol similar to the one involved above in the investigation of the polarization texture with respect to the temperature. Namely, instead of variations of the temperature with a step $\delta t$, we consider variations of $U$ with a step $\delta U$ (see [12] for more details).

Fig. 5 presents the numerical results obtained from the parallelepipedic configuration, with $t=-0.9$ and $\delta U= \pm 0.001$. These results correspond to hysteresis loops. We have, by increasing the voltage potential from $U=-0.6$, the switchings schematized as below

$$
(M P) \underset{U=0.062}{\longrightarrow}(C P) \underset{U=0.314}{\longrightarrow}(M P) .
$$

These abrupt switchings are irreversible, and define a hysteresis branch. By decreasing the voltage potential, from (MP) and $U=0.6$, we observe the switchings represented as follows

$$
(M P) \underset{U=-0.062}{\longrightarrow}(C P) \underset{U=-0.314}{\longrightarrow}(M P),
$$

defining the second hysteresis branch, in a symmetric way to the first branch as regards the origin. Thus, we get a hysteresis loop. Similarly, by decreasing the voltage potential from (CP) and $U \gtrsim 0.062$, the switching schematized as below arises

$$
(C P) \underset{U=-0.052}{\longrightarrow}(M P) \text {. }
$$

This is an abrupt switching, leading us to underline the existence of another hysteresis loop, called the "local hysteresis loop" here and in the next subsections. It arises also a second local hysteresis loop, symmetric to the previous one as regards the origin. It follows then, in particular, the existence of several distinct states when $U=0$; such a multidomain switching opens new routes for the design of high-capacity nanosize memory-storage devices in the ferroelectric-based nanoelectronics [13].

Similar results were obtained from $\mathcal{T}_{h}^{(1)}$; in particular, it arises also from this configuration a "global" hysteresis loop and two local hysteresis loops. 
Let us indicate that the numerical results obtained here do not deteriorate from the considerations of some larger mesh sizes, in the cylindrical frame as well as in the parallelepipedic context.

\subsection{Calibration effects}

We are here concerned with the numerical study of the influence of certain parameters of the ferroelectric model by inspecting also how the associated critical temperature can be adjusted. Let us already mention in particular that, as regards the geometrical parameters, the related variations will not involve numerical instabilities, although the framework developed in [1] may remedy such a situation as indicated in the previous subsection. Of course, although sufficiently small when compared to the size of the medium $\Omega$, the size of the ferroelectric layer will not however appear as too small in these variations that are in accordance with the applications context.

\subsubsection{Influence of the parameters on the state switchings}

We investigate here the influence of the size of the ferroelectric layer and of the permittivity of the paraelectric environment, as regards the state switchings. This study is based on variations of the geometrical parameter represented by the width, $R_{f}$, of the ferroelectric layer as well as on variations of the physical parameter $\varepsilon_{p}$ associated with the permittivity of the paraelectric environment. We focus on the description of investigations from states only related to the (MP) and (CP) profiles (which appear by an increasing of $U$ from low voltage potentials), in order to simplify the presentation hereafter.

The numerical results associated with Fig. 6(a) are obtained in the context of cylindrical geometries with respect to $R_{f}$, and in terms of $t$, by fixing $\varepsilon_{p}=90, U=0$, $\delta t=10^{-3}$. Several widths have been considered; namely, $R_{f}=1+k \delta r$, with $\delta r=$ $510^{-1}, 0 \leq k \leq 18$. Each consideration of $R_{f}$ corresponds then to a "cylindrical configuration" for which, systematically, the associated mesh size is approximately equal to 0.61 for $\bar{\Omega}$ and to 0.47 as regards $\overline{\Omega_{f}}$. Different situations occur. For small values of $R_{f}$, namely for the considerations defined as above and such that $R_{f} \leq 5.5$, only the (MP) profile exists. Then, for the ones which are such that $6 \leq R_{f} \leq 7.5$, the (CP) profile appears. A new type of switching occurs; namely, the switching of (MP) to (CP) is smooth. Regarding the considerations where $8 \leq R_{f} \leq 9.5$, the switching of (MP) to (CP) is abrupt. In each of these situations, the transition temperature, $t_{t}$, decreases while $R_{f}$ increases. For the largest value of $R_{f}$ related to these considerations, i.e., $R_{f}=10$, there exists only one profile; namely, the (CP) profile.

Fig. 6(b) presents, in the context of parallelepipedic geometries, the numerical results obtained from a similar study, with the same considerations for $R_{f}, \varepsilon_{p}, U$ and $\delta t$. Also, for each "parallelepipedic configuration", the associated mesh size is approximately equal to 0.61 as regards $\bar{\Omega}$ and to 0.47 about $\overline{\Omega_{f}}$. Similar phenomena occur: for these configurations where the width is such that $R_{f} \leq 5.5$, only the (MP) profile 


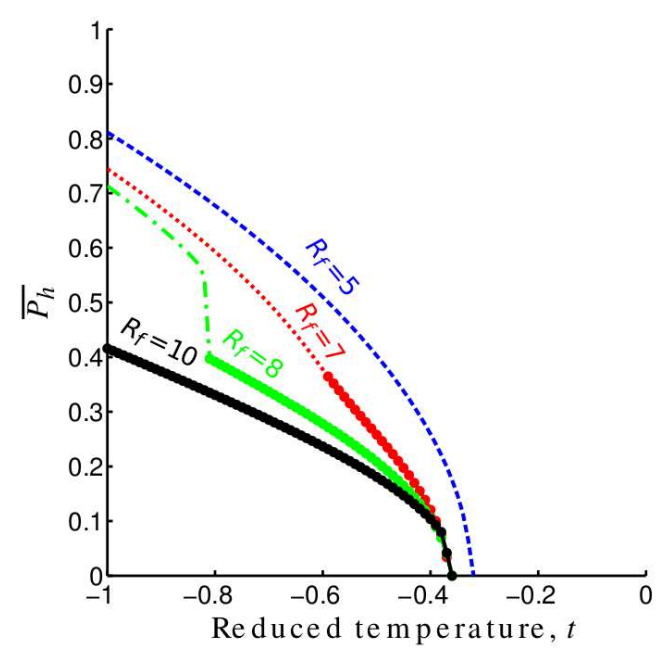

(a)

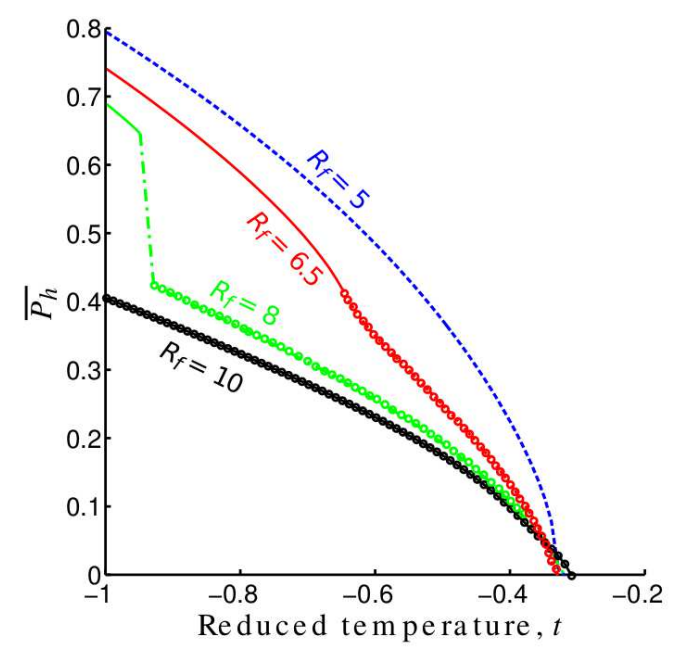

(b)

Figure 6: Representations of $\overline{P_{h}}$ with respect to the reduced temperature, for different values of $R_{f}$, from cylindrical configurations (a) and parallelepipedic configurations (b). The mark "o" denotes states associated with the (CP) profile, and the absence of this mark concerns states associated with the (MP) profile.

appears and then, for $R_{f} \in\{6,6.5\}$, (MP) switches continuously to (CP). For the considerations where $7 \leq R_{f} \leq 8$, (MP) switches abruptly to (CP) and for the ones such that $R_{f} \geq 8.5$, only the (CP) profile exists.

Based on the same variations of $R_{f}$ and for the same associated meshes, we have investigated the existence of hysteresis loops from the cylindrical context, with $t=$ -0.9 and $\delta U= \pm 10^{-3}$. Fig. 7(a) presents the numerical results deriving from this investigation. Only abrupt transitions are obtained, and the (CP) profile does not exist for any choice of $R_{f}$. There exist two types of loops:

- for the considerations such that $R_{f} \leq 5.5$, there exists a switching of a state, associated with the (MP) profile, to another one. Here, we denote by (1H-1P) this type of obtained curve, in which only one hysteresis loop and a unique kind of profile appear;

- for the considerations where $R_{f} \geq 6$, jumps are obtained and schematized as below

$$
(M P) \underset{U=U^{\star}}{\longrightarrow}(C P) \underset{U=U^{\star \star}}{\longrightarrow}(M P),
$$

where $U^{\star}$ and $U^{\star \star}$ are two values, related namely to $R_{f}$. These are abrupt transitions, and there exist three hysteresis loops (the two local hysteresis loops that arise in addition are not represented in Fig. 7(a) in order to not overload it). We denote by $(3 \mathrm{H})$ this type of loops.

Similar results deriving from the parallelepipedic context, with the same considerations for $R_{f}, t$ and $\delta U$, are represented in Fig. 7(b). Again, only smooth transitions and two types of hysteresis curves are obtained: 


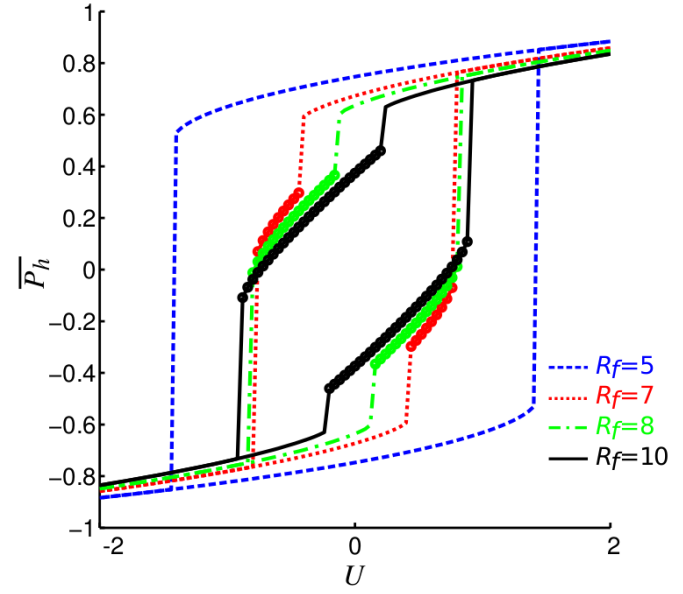

(a)

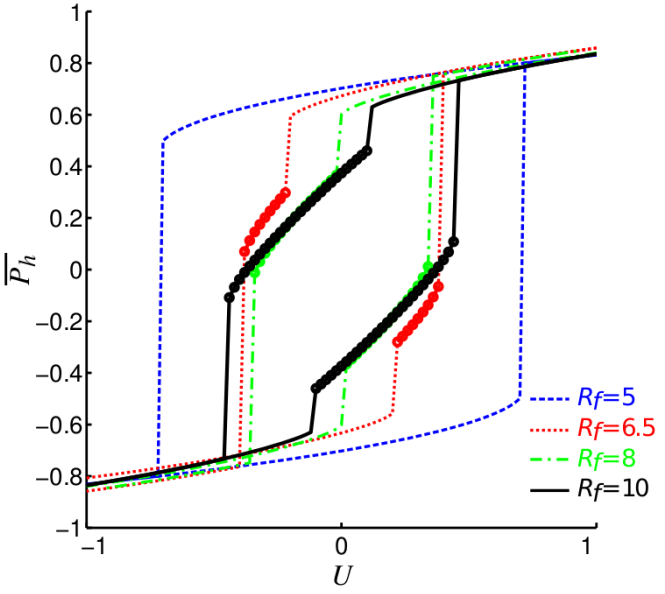

(b)

Figure 7: Hysteresis loops obtained for different values of $R_{f}$, with $t=-0.9$, from cylindrical configurations (a) and parallelepipedic configurations (b). The mark " $\circ$ " denotes states associated with the (CP) profile, and the absence of this mark concerns states associated with the (MP) profile.

- for the considerations such that $R_{f} \leq 5.5$, only the (MP) profile exists and we deal with (1H-1P) curves;

- whereas for the ones corresponding to $R_{f} \geq 6$, the (MP) and (CP) profiles appear, so we get $(3 \mathrm{H})$ curves.

Let us now study the influence of the physical parameter $\varepsilon_{p}$. We consider a finite sequence of values, $\varepsilon_{p}=10+k \delta \varepsilon$, with $\delta \varepsilon=10,0 \leq k \leq 19$. Also, we fix $R_{f}=8$ for the cylindrical configuration, and set $R_{f}=7.09$ for the parallelepipedic configuration in order to deal with ferroelectric layers of similar volumes in the two cases.

Fig. 8(a) presents, in the cylindrical context, the behavior of $\overline{P_{h}}$ with respect to $t$, by fixing $U=0$ and $\delta t=10^{-3}$. As previously in the study of variations of $R_{f}$, four different situations occur. For the considerations of $\varepsilon_{p}$ such that $\varepsilon_{p} \leq 70$, only the (CP) profile exists, then for the ones where $80 \leq \varepsilon_{p} \leq 110$, (MP) switches abruptly to (CP) whereas for the ones such that $120 \leq \varepsilon_{p} \leq 160$, this switching is smooth. Finally, for $\varepsilon_{p} \geq 170$, there exists only the (MP) profile.

Fig. 8(b) presents, from the parallelepipedic context, the numerical results obtained from a similar study, with the same considerations for $\varepsilon_{p}, U$ and $\delta t$. The four situations described above occur also. For the considerations where $\varepsilon_{p} \leq 70$, there exists only the (CP) profile. For $\varepsilon_{p} \in\{80,90,100\}$, the (MP) profile switches abruptly to (CP), then, for the considerations such that $110 \leq \varepsilon_{p} \leq 160$, the switching is smooth between these two profiles, and finally, when $\varepsilon_{p} \geq 170$, only the (MP) profile exists.

Fig. 9(a) presents, in association with these variations of $\varepsilon_{p}$, the results arising from $\mathcal{T}_{h}^{(1)}$ and regarding the investigation of the existence of hysteresis loops, with $t=-0.9$ and $\delta U=10^{-3}$. Two new types of curves arise in addition to the two previous ones: 


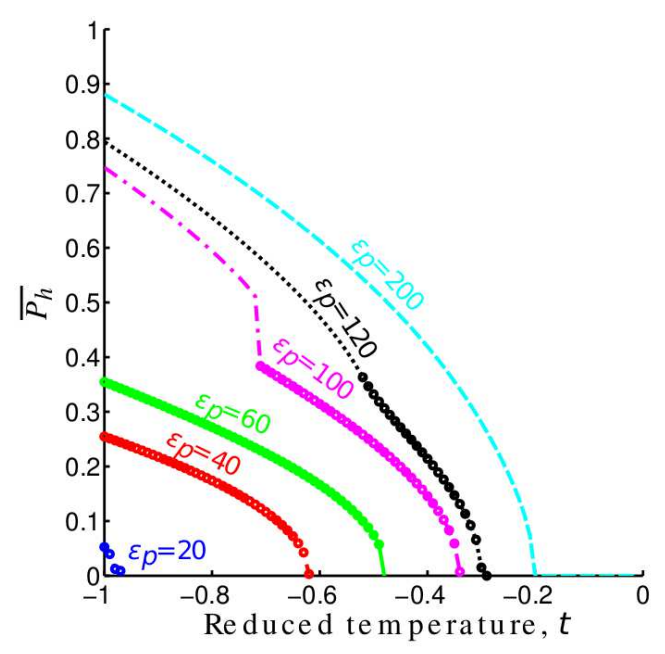

(a)

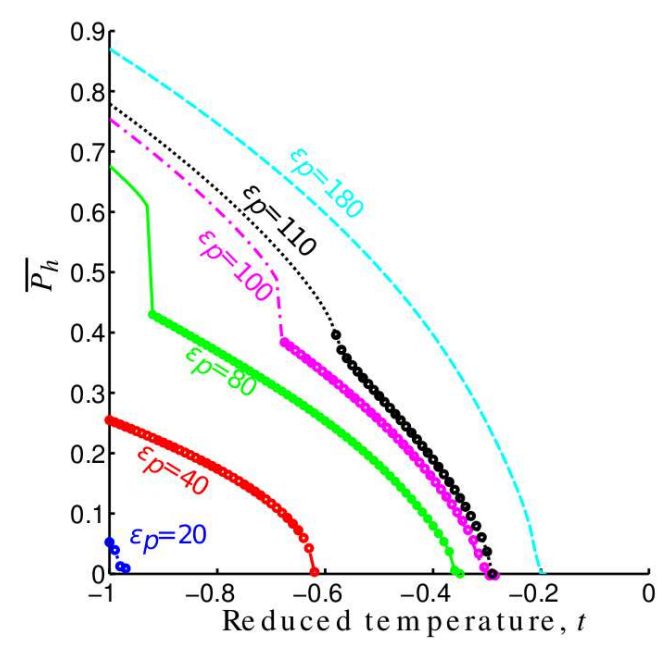

(b)

Figure 8: Representations of $\overline{P_{h}}$ with respect to the reduced temperature, for different values of $\varepsilon_{p}$, from $\mathcal{T}_{h}^{(1)}(\mathrm{a})$ and $\mathcal{T}_{h}^{(2)}$ (b). The mark "o" denotes states associated with the $(\mathrm{CP})$ profile, and the absence of this mark concerns states associated with the (MP) profile.

- for $\varepsilon_{p} \in\{10,20\}$, the transitions schematized as below, where $U_{\star}$ and $U_{\star \star}$ are two values related namely to $\varepsilon_{p}$,

$$
(M P) \underset{U=U_{\star}}{\longrightarrow}(C P) \underset{U=U_{\star \star}}{\longrightarrow}(M P)
$$

are smooth. As a consequence, no hysteresis loop appears, and we denote by $(\mathrm{OH})$ this type of curve. This is a limiting case;

- for $\varepsilon_{p} \in\{30,40,50\}$, the (MP) and (CP) profiles appear and the first transition $(M P) \rightarrow(C P)$ is smooth, whereas the second one, from a state associated with (CP) to another one, is abrupt. Thus, there exists one hysteresis loop involving two profiles. We denote by $(1 \mathrm{H}-2 \mathrm{P})$ this type of curve;

- for the considerations where $60 \leq \varepsilon_{p} \leq 130$, the two transitions schematized as follows, $(M P) \rightarrow(C P) \rightarrow(M P)$, are abrupt. The type of $(3 \mathrm{H})$ curves arises here also;

- for the considerations corresponding to $\varepsilon_{p} \geq 140$, the transition from a state associated with (MP) to another one appears and we get again (1H-1P) curves.

As indicates Fig. 9(b), similar results arise from $\mathcal{T}_{h}^{(2)}$, for $t=-0.9$ and $\delta U=10^{-3}$. Namely, for $\varepsilon_{p} \in\{10,20,30\},(\mathrm{OH})$ curves are obtained, while the considerations $\varepsilon_{p} \in$ $\{40,50,60\}$ correspond to (1H-2P) curves; the considerations where $70 \leq \varepsilon_{p} \leq 110$ lead to $(3 \mathrm{H})$ curves and the ones such that $\varepsilon_{p} \geq 120$ provide $(1 \mathrm{H}-1 \mathrm{P})$ curves. 


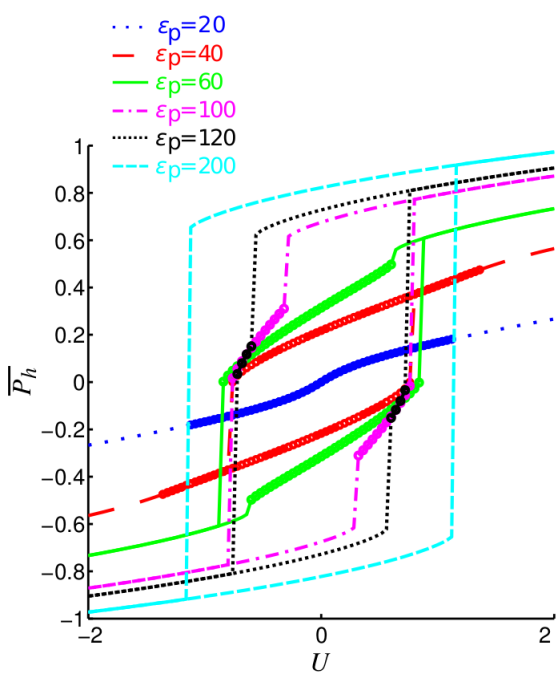

(a)

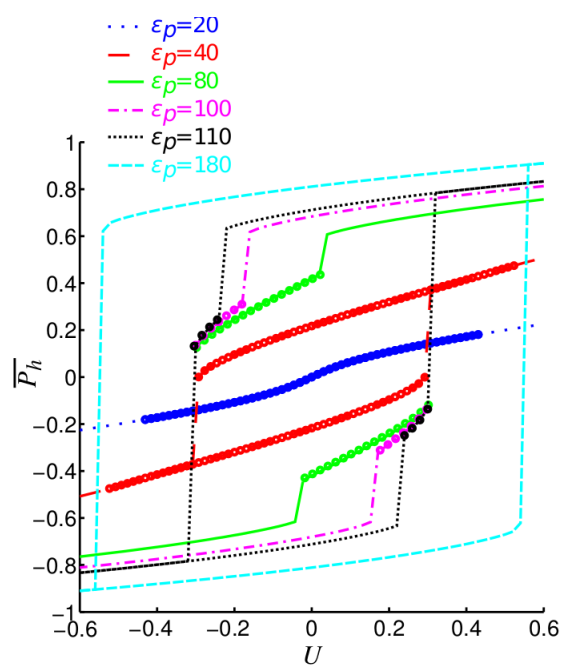

(b)

Figure 9: Hysteresis loops obtained for different values of $\varepsilon_{p}$, with $t=-0.9$, from $\mathcal{T}_{h}^{(1)}$ (a) and $\mathcal{T}_{h}^{(2)}$ (b). The mark "o" denotes states associated with the (CP) profile, and the absence of this mark concerns states associated with the (MP) profile.

Independently of the considered configurations, the same types of transitions under the influence of the geometrical parameter $R_{f}$ are retrieved also under the influence of the physical parameter $\varepsilon_{p}$. Thus, if we deal with a small ferroelectric layer, the behavior corresponding to the one of a larger layer can be obtained by acting on the permittivity of the paraelectric environment: we are then concerned with a kind of material doping. An aspect is that all the properties are not however perfectly preserved, since the critical temperature seems to depend strongly on $\varepsilon_{p}$ and much less on $R_{f}$; an action on this aspect will be investigated later.

We observed that the same diversity of classes of hysteresis loops was not found from variations of $R_{f}$; namely, $(\mathrm{OH})$ and $(1 \mathrm{H}-2 \mathrm{P})$ curves were not found. Let us consider other values of the temperature in order to prospect more.

Fig. 10 concerns the results arising from $\mathcal{T}_{h}^{(1)}$ and regarding the investigation of the existence of hysteresis loops, with $\varepsilon_{p}=90$ and $\delta U=10^{-3}$, for several values of the temperature; namely, $t=-0.05-k \delta t$, with $\delta t=10^{-1}, 0 \leq k \leq 9$. For these considerations, we find the three types of curves described before. In the case where $t \geq-0.25$, only the (MP) profile appears, so we obtain $(\mathrm{OH})$ curves. This result was expected since it is found before that, for these values of parameters, the critical temperature is -0.318 ; so, the material system is subject to its paraelectric phase. In the case where $t=-0.35$, it is subject to its ferroelectric phase and the two profiles (MP) and (CP) exist: the switching between these is smooth, and we thus obtain the type of $(\mathrm{OH})$ curves again. Then, for the considerations where $-0.65 \leq t \leq-0.45$, the switching between (MP) and (CP) is abrupt, and we thus have (1H-2P) curves. Finally, for $t \in\{-0.75,-0.85,-0.95\}$, all the switchings are abrupt, and we obtain (3H) curves. 


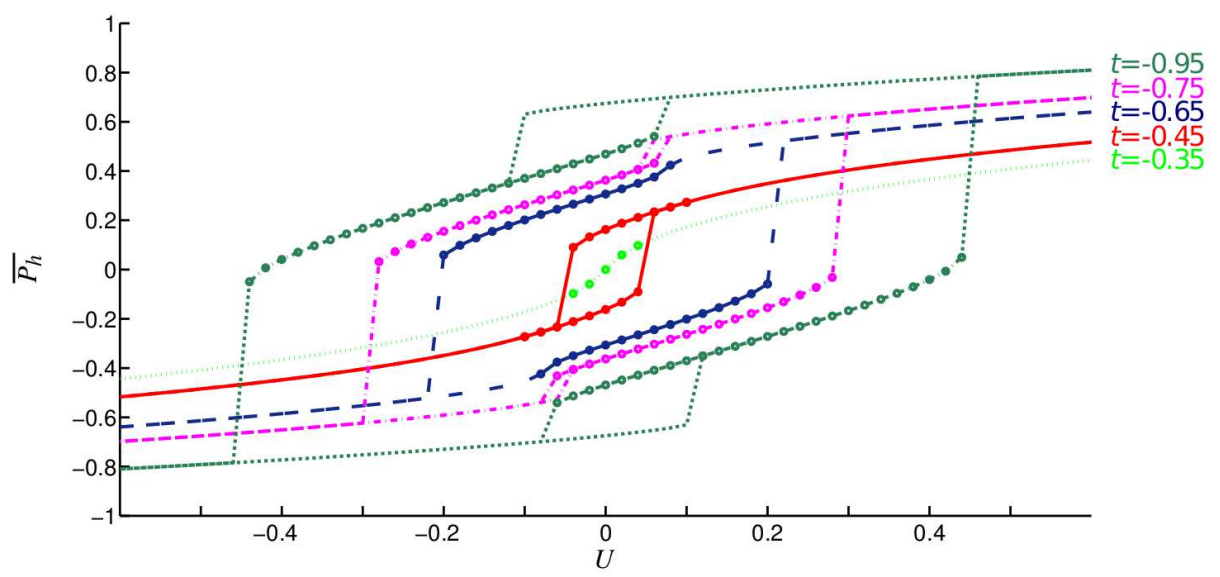

Figure 10: Hysteresis loops obtained for different values of $t$, from $\mathcal{T}_{h}^{(1)}$. The mark " $\mathrm{o}$ " denotes states associated with the (CP) profile, and the absence of this mark concerns states associated with the (MP) profile.

We are thus concerned with the same diversity of classes of hysteresis loops as in the frame of the variations of $\varepsilon_{p}$.

\subsubsection{Calibration of the critical temperature}

We have observed in particular previously that the permittivity of the paraelectric environment seems to have a strong influence on the critical temperature of the material system (see, e.g., Fig. 8). A similar influence was not noted from the variations of the width of the ferroelectric layer (see, e.g., Fig. 6).

Here, we want to study how precisely the critical temperature, $t_{c}$, behaves from common variations of the paraelectric environment permittivity and of the height, $H_{f}$, of the ferroelectric layer. For this, we consider several values of the pair $\left(\varepsilon_{p}, H_{f}\right)$. Namely, we set $\varepsilon_{p}=1+k$, with $0 \leq k \leq 9$, as well as $\varepsilon_{p}=10+10 k$, with $0 \leq k \leq 19$, and take $H_{f} \in\{1.1,1.4,1.7,2,2.25,2.5\}$, in the parallelepipedic context by fixing again $R_{f}=7.09$. For each consideration of $\left(\varepsilon_{p}, H_{f}\right)$, the behavior of the system with respect to the temperature is studied. Each of the profiles obtained has a critical temperature, determined by fixing also $\delta t=10^{-2}$, and it is of course retained that the largest one of these temperatures denotes the critical temperature, $t_{c}$, of the system. Let us specify that, systematically, the critical temperature related to the (P2B) profile, existing in each of the achieved experiments, is the largest one.

The obtained results are represented in Fig. 11, where the critical temperature is reported with respect to $\varepsilon_{p}$ and $H_{f}$. It derives that calibrations can be performed: for instance, for reaching the critical temperature $t_{c}=-0.5$, when the height of the ferroelectric layer is such that $H_{f}=2.5$, it is necessary to have for the paraelectric environment a permittivity such that $\varepsilon_{p} \approx 31$. It is also possible to reach the same critical temperature when the ferroelectric layer height is such that $H_{f}=1.4$, by considering 


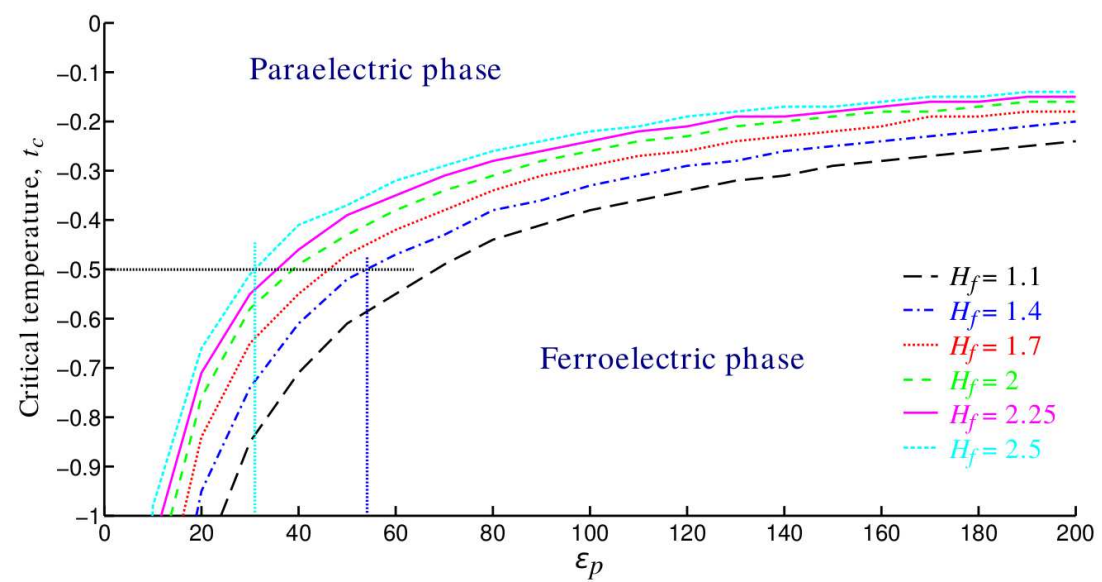

Figure 11: Numerical variation of the critical temperature with respect to $\varepsilon_{p}$, for different values of $H_{f}$ from the parallelepipedic configurations, and with $U=0$.

now a paraelectric environment for which the permittivity is such that $\varepsilon_{p} \approx 54$.

We find that $t_{c}$ depends on $\varepsilon_{p}$, but there is moreover a dependence on $H_{f}$ : the critical temperature increases with respect to the height $H_{f}$. The results represented in Fig. 11 indicate that $\varepsilon_{p} \mapsto t_{c}\left(\varepsilon_{p}\right)$ increases for $\varepsilon_{p}$ taking large values, independently of the considered values for $H_{f}$. Also, it occurs that $t_{c}$ increases slowly with respect to these values of $H_{f}$ and for each of the large values of $\varepsilon_{p}$. Similar results persist when we reproduce entirely the study by taking now for instance $R_{f}=8$. In particular, this underlines the aspect already reported and relating that the parameter $R_{f}$ does not have a strong influence on the critical temperature.

Let us mention that similar results derive also from numerical experiments achieved in the context of cylindrical configurations.

\section{Concluding remarks and perspectives}

From a variational method based on the Ginzburg-Landau formalism in combination with the electrostatics equations that was introduced in [11], we have numerically investigated here the switching behavior aspects and calibration effects relative to finite media embedding fully a three-dimensional ferroelectric layer in a paraelectric environment. The associated discrete nonlinear system, deriving from finite element discretizations, is solved with the help of a described iterative approach that combines two inexact Newton techniques. The first one, globalized with a linesearch method, converges slowly for a large choice of initializations, whereas the second one, more standard, converges faster but for a restricted choice of initializations.

The numerical investigations were performed by involving platelet samples in parallelepipedic and cylindrical configurations. In these investigations, the study of the influence of geometrical and physical parameters was based on variations of the width of the layer as well as on its height, and on variations of the paraelectric environment 
permittivity. Two switching types, namely related to smooth or abrupt state switchings, as well as various hysteresis curves have been found. A particular property highlighted here is that, independently of the considered configurations, a same switching behavior under the influence of the ferroelectric layer width is retrieved from the influence of the paraelectric environment permittivity. Namely, if we deal with a ferroelectric layer of small size, the behavior corresponding to the one of a larger width can be retrieved by acting upon the paraelectric environment permittivity. Moreover, and in particular, we studied the behavior of the transition temperature, between the paraelectric and ferroelectric phases, with respect to the paraelectric environment permittivity and to the ferroelectric layer height. It is observed that by dealing with a ferroelectric layer of small size, such a temperature in correspondence with a thicker layer can be retrieved by acting upon the paraelectric environment permittivity. In particular, the associated findings deriving from these investigations would aim at impregnating physical experiments that could be achieved for instance with samples of Sodium Nitrite in the design of the ferroelectric layer.

The perspectives of this work consider numerical investigations in the time dependent context, by dealing again with a fully embedded ferroelectric layer. This context shall require a model involving a three-dimensional physical region of interest which, in contrast when compared for instance with the one studied from a theoretical point of view in [2], is not represented alone by a ferroelectric material. These investigations shall concern in particular the study of state switchings as well as of the existence of hysteresis loops, also under the influence of the geometrical and physical parameters, without excluding the long time consideration too.

\section{References}

[1] H. Ammari, L. Halpern AND K. HAmdache, Asymptotic behaviour of thin ferromagnetic films, Asymptot. Anal., 24 (2000), pp. 277-294.

[2] H. AMmARI AND K. HAMDACHE, Global existence and regularity of solutions to a system of nonlinear Maxwell equations, J. Math. Anal. Appl., 286 (2003), pp. 51-63.

[3] E. V. ChENSKII AND V. V. TARASEnKo, Theory of phase transitions into inhomogeneous states in organic ferroelectrics in an external electric field, Zh. Eksp Teor. Fiz, 83 (1982), 1089. [Sov. Phys. JETP, 56 (1982), 618.]

[4] R. S. DEMbo, S. C. EISENStAT AND T. SteiHAug, Inexact Newton methods, SIAM J. Numer. Anal., 19 (1982), pp. 400-408.

[5] V. L. Ginzburg, Polarization and piezoelectric effect in BaTiO3 near the ferroelectric transition point, Zh. Eksp. Teor. Fiz, 19 (1949), 36.

[6] P. GRISVARD, Elliptic Problems in Nonsmooth Domains, Pitman Advanced Pub. Program Boston, 1985.

[7] C. KITTEL, Theory of the structure of ferromagnetic domains in films and small particles, Phys. Rev., 70 (1946), 965.

[8] L. D. Landau And E. M. Lifshitz, Course of Theoretical Physics - Electrodynamics of Continuous Media, vol. 8, Elsevier, 1985.

[9] L. D. LANDAU AND E. M. LIFSHITZ, On the theory of the dispersion of magnetic permeability in ferromagnetic bodies, Phys. Z. Sowjet., 8 (1935), 153. 
[10] I. A. Luk'yanchuk, L. LAHoche AND A. SenÉ, Universal properties of ferroelectric domains, Phys. Rev. Lett., 102 (2009).

[11] P.-W. MARTELLI, Modélisation et simulations numériques de la formation de domaines ferroélectriques dans des nanostructures 3D, Ph.D. Thesis, Université de Lorraine, 2016.

[12] P.-W. Martelli and S. M. Mefire, Numerical modeling and investigations of 3D devices with ferroelectric layer fully embedded in a paraelectric environment, Computers and Mathematics with Applications, 76 (2018), pp. 451-470.

[13] P.-W. Martelli, S. M. Mefire And I. A. Luk'yanchuk, Multidomain switching in the ferroelectric nanodots, Europhysics Lett., 111 (2015), 50001.

[14] V. N. NechaEV AND A. V. Viskovatykh, On the change in the mechanism of the phase transition in a ferroelectric inclusion of a ferroelectric-dielectric nanocomposite as a function of its size, Phys. Solid State, 57 (2015), 722.

[15] J. Nocedal AND S. J. WRIGHT, Numerical Optimization, Springer, 2006.

[16] Y. SAAD AND M. H. SCHULTZ, GMRES: A generalized minimal residual algorithm for solving nonsymmetric linear systems, SIAM J. Sci. Stat. Comput., 7 (1986), pp. 856-869.

[17] Y. SHIOZAKI, E. NAKAMURA AND T. Mitsui, Ferroelectrics and Related Substances. Oxides Part 1: Perovskite-type Oxides and $\mathrm{LiNbO}_{3}$ Family, Springer-Verlag, 1981.

[18] R. C. Sмith, Smart Material Systems, SIAM, Philadelphia, 2005.

[19] S. K. Streiffer, J. A. Eastman, D. D. Fong, C. Thompson, A. Munkholm, M. V. RAmana Murty, O. Auciello, G. R. BAi AND G. B. StePhenson, Observation of nanoscale $180^{\circ}$ stripe domains in ferroelectric PbTiO3 thin films, Phys. Rev. Lett., 89 (2002).

[20] A. H. STROud, Fifth degree integration formula for the n-simplex, SIAM J. Numer. Anal., 6 (1969), pp. 90-98.

[21] P. WolfE, Convergence conditions for ascent methods, SIAM Review, 11 (1969), pp. 226235. 